\title{
Paying to Match: Decentralized Markets with INFORMATION FRICTIONS*
}

\author{
Marina Agranov ${ }^{\dagger}$ Ahrash Dianat ${ }^{\ddagger}$ Larry Samuelson ${ }^{\S}$ Leeat Yariv $^{\mathbb{I}}$
}

May 11, 2022

\begin{abstract}
We experimentally study decentralized one-to-one matching markets with transfers. We vary the information available to participants, complete or incomplete, and the surplus structure, supermodular or submodular. Several insights emerge. First, while markets often culminate in efficient matchings, stability is more elusive, reflecting the difficulty of arranging attendant transfers. Second, incomplete information and submodularity present hurdles to efficiency and especially stability; their combination drastically diminishes stability's likelihood. Third, matchings form "from the top down" in complete-information supermodular markets, but exhibit many more and less-obviously ordered offers otherwise. Last, participants' market positions matter far more than their dynamic bargaining styles for outcomes.
\end{abstract}

Keywords: Matching Markets, Transfers, Incomplete Information, Experiments.

JEL: C78, C92, D02, D47.

\footnotetext{
${ }^{*}$ We thank Jeongbin Kim and Pellumb Reshidi for superb research assistance. We gratefully acknowledge financial support from the International Foundation for Research in Experimental Economics and from the NSF, through grant SES 1629613.

${ }^{\dagger}$ Division of the Humanities and Social Sciences, California Institute of Technology, Email: magranov@hss. caltech. edu ‡Department of Economics, University of Essex, Email: a.dianat@essex.ac.uk

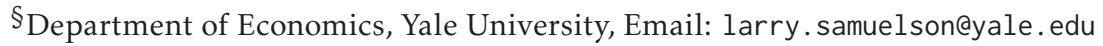

${ }^{\mathbb{I}}$ Department of Economics, Princeton University, Email: lyariv@princeton.edu
} 


\section{Introduction}

\subsection{Overview}

A large and successful literature studies matching markets with complete information. It assumes individuals know not only their own preferences over possible allocations, but also all others' preferences. This assumption is often made for tractability and is reasonable for some applications. In large school choice systems, for example, historical data on admission patterns might be persistent and therefore predictable. Nevertheless, there are numerous applications in which incomplete information regarding others' preferences, at times even one's own, might be present. Newly-minted doctors seeking a residency in a specific specialty may not be privy to the idiosyncratic preferences of both their peers and the hospitals they interview with. Similarly, fresh academic Ph.D.'s may not be fully informed of other applicants' preferences or of universities' particular research and teaching needs. Outside the labor market, potential adoptive parents are unlikely to know the preferences over child characteristics of other potential adoptive parents seeking children, or of the birth mothers relinquishing them. In marriage markets, an entire consulting industry builds on the premise that individuals frequently misread others' preferences, even after lengthy interactions. While incomplete information is prevalent across these applications, there is a paucity of empirical data to elucidate its impacts.

We report results from an array of lab experiments in which participants match through decentralized interactions, as in the examples above. Our treatments vary the surplus structure and the initial information available to market participants about others' preferences. We illustrate the substantial effect limited information has on outcomes and the regularities it produces.

Since many markets operate through transfers-labor markets often entail targeted wages, housing markets generate exchanges via prices, etc.-we consider decentralized matching markets allowing for transfers. While common wisdom suggests that transfers assist in equilibrating economic systems, they also allow for various bargaining frictions to emerge when negotiations are unconstrained. Differences in bargaining styles, failures to follow up with appropriate offers, and the like could, in principle, affect the generated match surpluses and their split between parties. Furthermore, in practice, implementing the "right" transfers might be challenging: agents often interact with a small set of potential partners and have limited information on what others 
should reasonably expect to receive. The challenge of finding the "right" transfers is compounded by agents' incomplete information about others' preferences. Our design allows us to discern how transfers evolve and get determined.

Experimental Design. While our study speaks to many applications, field data are difficult to gather. Rarely do we know market participants' true preferences, their detailed interactions, or the information available to them. The lab setting allows us to control for different market features and observe how individuals respond.

We consider two-sided one-to-one markets in which agents can make match offers to one another. Agents in the market are characterized by their "types," which can stand for education level or expertise in labor markets, age in child-adoption processes, or pizzazz in the marriage setting. All agents prefer to be matched with higher-type agents: they generate a greater match surplus.

The marginal benefit from pairing with a higher-type individual varies across our experimental markets. Specifically, we consider two surplus structures: supermodular and submodular. In supermodular markets, higher types experience a greater marginal increase in generated surplus from matching with higher types. Consequently, the utilitarian efficient matching is positively assortative. In submodular markets, the reverse holds: it is the lower-type agents who experience the greatest marginal benefit from increasing their partner's quality. In such markets, utilitarian efficiency implies negatively assortative matchings. Going back to Becker (1973), the matching literature has often assumed match surpluses exhibit either supermodularity or submodularity. Nonetheless, the empirical literature is largely silent on which of the two classes is relevant for various applications. As we show, the distinction between the two is crucial for outcomes.

In our experimental markets, interactions are decentralized: individuals can make offers to one another in an unscripted fashion, with the sole restriction that only one offer can be made or accepted at any time. An offer indicates a split of the match surplus. Accepted offers generate tentative matches - they can be broken by making a different offer that gets accepted, or by accepting an offer. A market terminates after 30 seconds of inactivity.

In our complete-information treatments, participants know the surplus generated by each pair of participants in the market. In our incomplete-information treatments, participants enter the market knowing the profile of match surpluses they can generate. That is, they know their own type. They do not, however, know the types of other participants. In particular, at the start of the 
market, they do not know who will help them generate which surplus. Learning occurs through market interactions. Whenever an offer is accepted, both partners learn the match surplus they jointly generate and the resulting payoffs they receive. ${ }^{1}$ The full market observes the creation of matches, as well as the surplus that recipients of accepted offers receive, but not the total surplus such matches generate.

Empirical Regularities. Several insights emerge from our analysis. As we describe in our literature review below, prior experimental work supports the impression that, at least without transfers, decentralized interaction yields stable outcomes. Our results show that matching markets with transfers often produce utilitarian efficient matchings, but stable outcomes are less common. $^{2}$ Thus, there is a sense in which the common wisdom on the benefits of transfers holds water in our data, albeit at the cost of stability. This effect is particularly salient when the surplus is submodular. Specifically, with complete information, $94 \%$ of our supermodular markets culminate in efficient, positively assortative outcomes and $78 \%$ of market results are stable. When preferences are submodular, only $73 \%$ of our markets yield efficient, negatively assortative outcomes and a mere $14 \%$ of ultimate outcomes are stable.

When information is incomplete, the theoretical literature offers little guidance as to the dynamics and outcomes one might expect; see our review of the literature. In particular, there is little work inspecting the information revealed through the operations of a market that starts with incomplete information. In simple incomplete-information markets such as ours, where participants enter with considerable information and every accepted or rejected offer yields more information, interactions could plausibly lead to complete information. Therefore, throughout much of our analysis, we use complete-information stability as a benchmark, returning to this issue in Section 5.1.

In our data, incomplete information and submodularity constitute substantial obstacles to both efficiency and stability. Indeed, with incomplete information, in markets with a supermod-

\footnotetext{
${ }^{1}$ Offers still entail the specification of a transfer. Absent information on match surpluses, certain offers can lead to negative payoffs. We allow participants to cancel such offers ex-post. We do not see participants "gaming" the experiments through this option.

${ }^{2}$ Utilitarian efficient matchings maximize the sum of match surpluses. Stability with transfers corresponds to a matching and a profile of transfers such that no pair of agents can find a transfer that, if implemented, would make their match desirable relative to what they already receive. It is well known that stable matchings are utilitarian efficient (e.g. Roth and Sotomayor, 1990).
} 
ular surplus structure, $84 \%$ of outcomes are efficient, while $54 \%$ are stable. With submodular surpluses, $39 \%$ of outcomes are efficient, and a mere 3\% are stable. In other words, the combination of incomplete information with submodularity nearly eliminates the likelihood of achieving stable outcomes. This observation opens the door for richer theories of matching with incomplete information: ones that allow for some market learning, but still yield outcomes that are not complete-information stable.

The analysis of offer dynamics provides a lens into how market outcomes are generated. When information is complete, markets governed by supermodular surpluses "match from the top," in the sense that high-type agents (who generate the greatest surplus) match first, followed by medium-type agents, and finally low-type agents. ${ }^{3}$ In contrast, the sequenceing of matches is more haphazard in submodular markets. Many more offers are made throughout those market operations. Early matches that "stick" are often between high-type agents, and generate a barrier to achieving market-wide efficiency. Medium-type agents follow suit, while low-type agents tend to match late, regardless of their partner's type.

When information is incomplete, regardless of the underlying surplus structure, initial matches are often transient and broken later in the market's operations as participants learn. This leads to far more market activity. Matches between incongruent types, low and high, occur earlier. Interestingly, the enhanced market activity we observe implies that, by the end of our markets' operations, most participants learn with whom they can generate which surplus, consistent with our focus on complete-information stability. However, even matches formed later in our incompleteinformation markets do not echo the patterns seen in their complete-information counterparts. Why do outcomes not mimic those in our complete-information markets? We show that our data are consistent with simple satisficing heuristics of participants. Based on their type, participants aim at a certain payoff and actively search for partners only below their satisficing threshold. This observation echoes evidence documented in individual decision-making; see Caplin, Dean, and Martin (2011). In our setting, satisficing emerges through dynamic market interactions.

A large literature, scientific and popular, suggests the importance of bargaining strategies in determining outcomes-for reviews, see Bertrand (2011) and Shell (2006). One may wonder whether our participants' individual tendencies help explain their obtained payoffs. We indeed

\footnotetext{
${ }^{3}$ This sequence resembles the Marshallian path studied by Plott, Roy, and Tong (2013).
} 
see large variation in individuals' bargaining styles, in terms of offer volume, number of matches created throughout a market, how demanding offers are, and the timing of interactions. Nonetheless, none of these has any substantial explanatory power for outcomes. Ultimately, market features and one's position in the market-namely, the type-appear to explain the lion's share of the payoffs participants receive. This result may be comforting to some: while individual characteristics and access to opportunities for self-improvement may greatly affect one's consequences, they do so only insofar as they alter one's position, or "quality," in the market. Once in the market, outcomes appear resilient to individual variations that go beyond participants' contributions to the surpluses they can generate.

Implications. Taken together, our results carry several important implications for market design. First, when transfers are available, stability through decentralized interaction is not guaranteed and depends crucially on the underlying preferences in the market. In particular, centralized clearinghouses may be especially beneficial when preferences are submodular. Second, incomplete information presents a substantial hurdle for achieving stability. Interventions directed at alleviating information frictions may therefore prove useful. Such interventions could entail allowing for more communication between participants, introducing public releases of information about market attributes, etc. Alternatively, centralization of the matching process may be particularly important when participants are not perfectly informed. The existing literature rarely considers clearinghouses with imperfectly-informed participants, and the consideration of those may be necessary. Last, educating participants to achieve their maximal potential "quality" as they enter a matching market may be of far greater importance than educating participants on how to negotiate their interactions once in the market.

\subsection{Related Literature}

A large theoretical literature considers matching markets with complete information, with or without transfers; see Roth and Sotomayor (1990). Incomplete information poses many challenges to the analysis of matching markets. Even the basic stability notion is difficult to translate when agents are not fully informed of everyone's preferences. Pairs that would block a matching with complete information-pairs of agents that prefer one another over their allocated match-may not form if agents cannot discern who would prefer a match with them. Furthermore, any at- 
tempt to form a blocking pair allows other participants to partially infer the preferences of agents involved, possibly others' when those are correlated.

Starting from Liu, Mailath, Postlewaite, and Samuelson (2014), several recent papers suggest stability notions for incomplete information markets, mostly restricting attention to incomplete information only on one market side. Bikhchandani (2017) considers matching markets without transfers. Liu et al. (2014), and relatedly Alston (2020) and Liu (2022), consider matching with transfers as we do. At the heart of the stability notions in these papers is the requirement that any agent's "deviation" from a matching, with its attendant transfers, ultimately leaves her (weakly) worse off. The stability notions in these papers are defined with respect to a given matching and a fixed specification of the uncertainty agents have about others' types. They do not consider the possibility that the match formation process may reveal information, rendering the specification of uncertainty endogenous and possibly leading to effectively complete information.

Stability notions are interesting due to the presumption that, in frictionless decentralized markets, agents would ultimately converge to a stable outcome. Nonetheless, only a few papers explicitly model the decentralized process that generates such stable outcomes. Roth and Vate (1990) offer non-strategic dynamics that yield stable outcomes, whereby at each stage, a random blocking pair is implemented. Complete information is implicitly assumed, as all blocking pairs are considered at every step. Ferdowsian, Niederle, and Yariv (2022) consider a decentralized market game in which firms and workers interact in a dynamic fashion. They illustrate the hurdles incomplete information or time frictions generate in establishing stability as a property of equilibrium outcomes. Chen and $\mathrm{Hu}(2020)$ focus on incomplete-information settings and construct an adaptive learning process leading to stable outcomes as they define them.

Hakimov and Kübler (2021) present a general overview of the experimental matching literature. Several papers in that literature share features with our design. ${ }^{4}$

Concurrent work by $\mathrm{He}, \mathrm{Wu}$, and Zhang (2022) considers decentralized matching with complete information, allowing for transfers between participants. He et al. (2022)'s design resembles that of our complete-information treatment, although their experimental markets have a prespecified time of operation. ${ }^{5}$ In line with our results, they observe far more stable outcomes in

\footnotetext{
${ }^{4}$ There are also several papers studying decentralized matching with complete information and no transfers, see Echenique and Yariv (2013) and references therein.

${ }^{5}$ In their design, participants whose match is broken in the last 15 seconds can make offers within 15 seconds.
} 
assortative than in anti-assortative markets. In contrast with our results, they observe a substantial fraction of unmatched individuals, potentially due to the limited-market horizon. Outside the matching context, Agranov and Elliott (2021) identify the important impacts of transfers on bargaining outcomes.

Nalbantian and Schotter (1995) report on experiments emulating the market for professional baseball players in the free-agent years. They analyze several allocation procedures with transfers, including a decentralized procedure in which agents are informed of their own bargaining outcomes, but not of others'. Pais, Pintér, and Veszteg (2020) study decentralized markets with multiple stable matchings. There are no transfers and markets operate over a fixed duration. In some treatments, participants are privately informed of their preferences. Incomplete information in their setting does not affect the stability or the efficiency of the final outcome. However, similar to what we observe, it boosts market activity. Niederle and Roth (2009) study unraveling by considering an incomplete-information setting in which one side of the market makes proposals to the other side over three experimental periods and information is exogenously and incrementally released. They illustrate the impact of proposal structure on matching outcomes and efficiency. Incomplete information has also been considered in centralized markets, mostly focusing on school-choice mechanisms and, therefore, absent transfers. See, for example, Chen and Sönmez (2006), Pais and Pintér (2008), and work that followed. ${ }^{6}$

\section{Experimental Protocol}

In our experiments, participants engage in a sequence of decentralized markets varying in two dimensions: the surplus structure, which is either supermodular or submodular, and the information available. In some matching markets, participants are fully informed of everyone's preferences, in others they receive only partial information. We describe each of these dimensions in turn. Sample experimental instructions are presented in Appendix C.

Our two-sided one-to-one matching markets are comprised of six participants, three on each side. Each participant has a role: a color or a food. These roles capture the market sides and can be metaphors for firms and workers in labor markets, men and women in the marriage market,

\footnotetext{
${ }^{6}$ There is also recent empirical work that highlights the consequences of incomplete information in school choice, see Kapor, Neilson, and Zimmerman (2020).
} 
Table 1: Match Surpluses (Efficient Allocation in Bold)

\begin{tabular}{lccc}
\hline \hline & Kiwi & Mango & Plum \\
\hline Blue & $\mathbf{8}$ & 16 & 24 \\
Crimson & 16 & 32 & 48 \\
White & 24 & 48 & $\mathbf{7 2}$ \\
\hline \hline
\end{tabular}

(a) Supermodular

\begin{tabular}{lccc}
\hline \hline & Kiwi & Mango & Plum \\
\hline Blue & 8 & 32 & $\mathbf{5 6}$ \\
Crimson & 32 & 48 & 64 \\
White & $\mathbf{5 6}$ & 64 & 72 \\
\hline
\end{tabular}

(b) Submodular

etc. There are three payoff-relevant types for each role. Specifically, the three color types are Blue, Crimson, and White. The three food types are Kiwi, Mango, and Plum. A matching specifies which colors and foods are unmatched, as well as a mapping between the remaining colors and foods that is one-to-one: if a color type, say Blue, is assigned to a food type, say Kiwi, then the reverse holds as well: Kiwi is assigned to Blue. ${ }^{7}$

Table 1 depicts the match surpluses governing preferences in our markets. Panel (a) corresponds to our supermodular surpluses, while panel (b) captures our submodular surpluses. In each, the entry corresponding to each color and food pair is the match surplus generated by that pair. Unmatched agents receive a payoff of 0 .

We consider two information structures. In our complete-information treatments, participants are fully informed of surpluses generated by all pairs in the market. In particular, they can readily rank their potential partners in terms of the surpluses they would jointly generate. In our incomplete-information treatments, participants know their own type: at the market's outset, they are informed of the three possible surpluses they can generate with the three potential partners on the other side of the market. However, they do not know which partner generates which surplus, nor which of the remaining two types have been assigned to which other agent on their side of the market. Formally, we permute the type labels in markets and inform participants only of their own type. The structure of payoffs is transparent, as well as the randomization procedure.

The general structure of each session is as follows. The governing match surpluses, either supermodular or submodular, as well as the information structure, are fixed throughout the session. Participants engage in one practice round, followed by 10 real rounds, each corresponding to a new market (of the same sort in terms of both preferences and information), with a freshly drawn random set of participants within the session. Participants maintain their role, a color or a food, throughout the session. However, in each round, they are randomly assigned one of the three

\footnotetext{
${ }^{7}$ In our experimental results, the incidence of unmatched participants is only $0.5 \%$ across all sessions.
} 
types corresponding to their role.

In our complete-information treatments, each participant observes the full surplus matrix throughout every round. ${ }^{8}$ In our incomplete-information treatments, each participant only observes her own possible match surpluses at the start of each round. ${ }^{9}$ As we soon explain, interactions in the market can reveal some information on who generates which surplus.

We now describe the rules of the matching protocol. In each round, participants start off unmatched. Each participant is free to make at most one match proposal to any individual of the opposite role at any given time. In the complete-information treatments, a match proposal specifies how the match surplus will be split among the two individuals-i.e., the proposer's payoff and the responder's payoff, summing up to the match surplus. In the incomplete-information treatments, a match proposal only specifies the responder's payoff, with the attendant proposer payoff revealed if the responder accepts. Thus, the proposer bears the payoff risk under incomplete information. This design choice was made to echo many applications in which the proposing side has limited information on the returns to her proposal. For instance, firms offering employment often cannot assess workers' abilities. Nonetheless, we limited the scope of risk by allowing proposers to immediately retract an offer if it turned out to generate a strict loss. For example, suppose Blue and Kiwi generate a match surplus of 8. If Blue offers Kiwi a payoff of 16, and Kiwi accepts, then Blue earns a payoff of -8 . Since Blue earns a negative payoff, we would allow her to unilaterally cancel the match. ${ }^{10}$ This design choice was made both for practical reasons, in order to limit the liability participants face, and also to mimic applications in which catastrophic relationships can be severed promptly. For instance, a worker who does not have their presumed credentials can be fired quickly by the firm that hired them in many labor-market settings. Whenever an offer that gives a positive payoff for both parties is accepted, the match can be dissolved only upon one of its participants making or receiving an alternative offer that is accepted.

A match generating a loss certainly conveys some information to the proposer. In general, whenever a match proposal is made and accepted, both the proposer and the responder observe

\footnotetext{
${ }^{8}$ To prevent participants from reacting to cosmetic features of the surplus matrix, we shuffled the rows and columns of the surplus matrix between rounds. In particular, specific colors and foods correspond to different match-surplus profiles in each round. As a result, the efficient matchings do not always coincide with the diagonal (in the positively assortative case) or anti-diagonal (in the negatively assortative case) of the surplus matrix.

${ }^{9}$ For example, Blue in panel (a) of Table 1 would know that her possible match surpluses are 8,16 , and 24 . However, she would not know which surplus corresponds to which match.

${ }^{10}$ In our data, $88 \%$ of negative offers were canceled by the proposers immediately. Other cases were associated with immediate new offers by the proposers. Either way, we never see negative payoffs for any participant at any round.
} 
their realized payoffs. Other participants observe the formation of the match and the responder's payoff. This is the main channel through which learning occurs under incomplete information.

In terms of market activity, participants are free to accept or reject match proposals they receive from other individuals. Any match proposal that is not accepted or rejected within 10 seconds automatically disappears and is effectively interpreted as a rejection. An acceptance forms a match. Participants who are currently matched can still make and receive new match proposals, with their current match persisting until such a new match proposal is accepted, at which point (only) the current match is dissolved. A round ends after 30 seconds of inactivity. In incompleteinformation treatments, in order to allow participants to avoid excessive waiting, we introduced a "move on to next round" button. Whenever at least 5 participants pressed the button, the market for that group and round terminated. ${ }^{11}$ A participant's payoff in their current match at the end of a round is their final payoff for that round. If a participant is unmatched at the end of a round, their final payoff is 0 for that round.

At the end of each session, participants also complete two risk-elicitation tasks and two altruism tasks. We use a version of the Gneezy and Potters (1997) investment task to measure risk preferences and a dictator game to measure altruism. ${ }^{12}$ All payoffs in the experiment are expressed in tokens. For the main part of the experiment, 10 tokens correspond to $\$ 1$. For the elicitation tasks, one token corresponds to $\$ 0.01$. Participants' total earnings in the experiment include a $\$ 10$ show-up payment, the sum of their final payoffs across the 10 rounds, and their payoffs from the auxiliary elicitation tasks.

The experimental sessions were run at the Experimental and Behavioral Economics Laboratory (EBEL) at UC Santa Barbara between July 2018 and October 2018. Each treatment, characterized by a combination of underlying preferences and information structure, was run in 4 sessions, each consisting of 12 participants. This generated 16 sessions overall and a total of 192 participants. Each session lasted approximately 90 minutes, and paid an average of $\$ 24.75$, in addition to a $\$ 10$

\footnotetext{
${ }^{11}$ We were particularly concerned about the sensitivity of our incomplete-information treatments to specific individuals prolonging rounds after long waits. Whenever 5 of the 6 group members are ready to halt market activity, we suspected the choice of the sixth individual to delay the end time would not affect outcomes. As will be seen, our incomplete-information treatments indeed took much longer in terms of both time and market activity.

${ }^{12}$ Specifically, in each of the two risk-elicitation tasks, participants were provided with 200 tokens that they had to allocate between a safe project, returning token for token, and a risky project. In the first task, each token invested in the risky project returned 2.5 tokens with $50 \%$ probability, and 0 otherwise. In the second task, each token invested returned 3 tokens with $40 \%$ probability, and 0 otherwise. Altruism was elicited using two dictator-game elicitations, one with an endowment of 100 tokens and another with an endowment of 200 tokens.
} 
show-up fee. The experiment was programmed and conducted with the oTree software (Chen, Schonger, and Wickens, 2016).

\section{Theoretical Preliminaries}

In both our supermodular and submoduar markets, types can be ordered. In Table 1, White generates higher surplus than Crimson, who generates higher surplus than Blue, for any food. Similarly, Plum generates higher surplus than Mango, who generates a higher surplus than Kiwi, for any color. Since labels of different types are shuffled across rounds, and the two sides of our markets are fully symmetric, we refer to the types generating the highest surplus as high types, those generating the intermediate surplus as medium types, and those generating the lowest surplus as low types.

Formally, let $C$ be the set of colors and $F$ the set of foods, with generic elements $f$ and $c$. Let $\pi: C \times F \rightarrow \mathbb{R}$ identify the surplus associated with each pair of agents. A matching (with no unmatched agents) is a bijection $m: C \cup F \rightarrow C \cup F$, where $m(C) \subseteq F, m(F) \subseteq C$, satisfying the restriction that if $m(c)=f$, then $m(f)=c$ for all $c \in C$ and $f \in F$. An outcome is a matching $m$ and a pair of transfer schemes $\tau_{C}: C \rightarrow \mathbb{R}$ and $\tau_{F}: F \rightarrow \mathbb{R}$.

Under complete information, an outcome $\left(m, \tau_{C}, \tau_{F}\right)$ is stable if it satisfies

$$
\begin{aligned}
\tau_{C}(c), \tau_{F}(f) & \geq 0 & & \forall(c, f) \in C \times F \\
\tau_{C}(c)+\tau_{F}(m(c)) & =\pi(c, m(c)) & & \forall c \in C \\
\tau_{C}(c)+\tau_{F}(f) & \geq \pi(c, f) & & \forall(c, f) \in C \times F .
\end{aligned}
$$

The first condition is an individual rationality requirement guaranteeing that no agent prefers to be unmatched. The second condition is a feasibility condition guaranteeing that transfers in a matched pair do not exceed the generated surplus. The third condition is a no-blocking condition that guarantees there is no pair of agents who can increase their transfers by matching with one another and appropriately dividing the attendant surplus. We say that a matching $m$ is stable if it is consistent with some stable outcome.

While all our treatments allow participants to make transfers to one another, it is useful to first contemplate a no-transfer benchmark, in which surpluses are divided at some fixed ratios 
between the two sides of the market. ${ }^{13}$ In this case, there is a unique stable matching in both the supermodular and submodular markets: the positive assortative matching (PAM) that matches individuals of the same type. In the markets depicted in Table 1, the unique stable matching would match the highest types, White and Plum, then the medium types, Crimson and Mango, and finally the low types, Blue and Kiwi.

When transfers are available, stable outcomes induce matchings that maximize the sum of surpluses: the utilitarian efficient matchings, hereafter referred to as simply efficient. For the two markets we consider, there may be many transfer profiles that sustain stable outcomes, see Appendix B for a formal characterization, but the matching itself is unique. In our supermodular markets, the matching maximizing utilitarian efficiency is the positive assortative matching (PAM). Furthermore, PAM together with transfers that split each surplus equally constitute a stable outcome. In our submodular markets, the matching maximizing utilitarian efficiency is the negative assortative matching (NAM). Importantly, there is no stable outcome in which each surplus is split equally. Instead, in the matches between low and high agents (Blue with Plum and White with Kiwi), the high agents (White and Plum) must receive more than half the surplus.

The existing theoretical literature offers less definitive guidance as to the behavior and outcomes one might expect under incomplete information. We adopt a permissive analog of completeinformation stability, deeming an outcome as unstable if there is a pair of agents with the property that (i) one of the agents can propose matching under terms that will make both agents better off, and (ii) the proposing agent knows there is no chance the proposal could make her worse off. To make this precise, for each color $c$, let the set $E_{c} \subset \mathbb{R}^{3}$, with generic element $(\pi(c, \cdot))$, contain vectors specifying the surpluses that $c$ can generate with each food $f$. Let $E_{f}$ be similarly defined for each food $f$. We say that the set $E_{c}$ (or $E_{f}$ ) is consistent with color c's (or food $f^{\prime}$ 's) experience if it excludes those values that the experience has revealed to be impossible. Then $\left(m, \tau_{C}, \tau_{F},\left(E_{c}\right)_{c \in C},\left(E_{f}\right)_{f \in F}\right)$ is incomplete-information stable if the sets $\left(E_{c}\right)_{c \in C},\left(E_{f}\right)_{f \in F}$ are consistent and

$$
\begin{aligned}
\tau_{C}(c), \tau_{F}(f) & \geq 0 & & \forall(c, f) \in C \times F \\
\tau_{C}(c)+\tau_{F}(m(c)) & =\pi(c, m(c)) & & \forall c \in C
\end{aligned}
$$

${ }^{13}$ That is, we can restrict $\tau(c)=\alpha \pi(c, m(c))$ and $\tau(f)=(1-\alpha) \pi(m(f), f)$ for all $c$ and $f$, where $\alpha \in(0,1)$. 


$$
\begin{array}{lll}
\tau_{C}(c)+\tau_{F}(f) \geq \min _{(\tilde{\pi}(c,)) \in E_{c}} \tilde{\pi}(c, f) & \forall c \in C \\
\tau_{C}(c)+\tau_{F}(f) \geq \min _{(\tilde{\pi}(\cdot, f)) \in E_{f}} \tilde{\pi}(c, f) & \forall f \in F .
\end{array}
$$

The first two conditions are the individual rationality and feasibility conditions. The latter two conditions are the no-blocking conditions, stated separately for colors and foods. For example, the no-blocking condition for colors asks color $c$ to identify the minimum surplus that could be generated with each food $f$, given what $c$ has learned. The condition guarantees that no split of that (pessimistic) surplus can leave both $c$ and $f$ better off. The last condition is the analog for foods.

If at least one of the participants in each possible match knows the surplus that would be generated by the match, then this notion of incomplete-information stability reduces to completeinformation stability. This sufficient condition will be met in our case if at least 5 market participants know the surplus they can generate with each opponent. Our results (Table 5 below) show that the average number of participants who learn from their direct experience-i.e., from matches in which they have at least temporarily participated-the surpluses they can generate with each opponent exceeds 5 . The matching process provides yet more information. We accordingly take complete-information stability as our benchmark throughout.

Despite the abundance of information participants accumulate through market interactions, we show that our incomplete-information markets do not routinely produce stable outcomes. In latter parts of the paper, we consider two explanations. First, market participants may make use of even less information than that which they have gained through their direct experience. Second, incomplete information may introduce glitches into the matching process beyond the lack of information-some offers a potential proposer knows impose no risk of a reduced payoff and benefit both parties involved, may not be made. In particular, participants may pursue decision criteria different from payoff maximization. Our analysis indicates the room for a richer theory of matching with incomplete information.

\section{Aggregate Outcomes}

We start by describing the aggregate outcomes in our experimental markets. We use all 10 rounds played in each session and omit the practice round. 


\subsection{Efficiency and Stability}

Table 2 depicts the fraction of efficient outcomes, those corresponding to a matching that maximizes the sum of match surpluses, i.e. the fraction of PAM for supermodular markets and NAM for submodular markets. The table also depicts the fraction of stable outcomes, consisting of both an efficient matching and a stable transfer profile. For the latter, we also display the fraction of outcomes that are "almost stable" in that the matching is efficient, and no pair can generate more than a small additional amount, 2 or 5 tokens, by blocking the observed outcome.

Table 2: Final Outcomes (allocations)

\begin{tabular}{ccccc}
\hline \hline & \multicolumn{2}{c}{ Complete Information } & \multicolumn{2}{c}{ Incomplete Information } \\
& $\begin{array}{c}\text { Supermodular } \\
\text { mean }(\mathrm{se})\end{array}$ & $\begin{array}{c}\text { Submodular } \\
\text { mean }(\mathrm{se})\end{array}$ & $\begin{array}{c}\text { Supermodular } \\
\text { mean (se) }\end{array}$ & $\begin{array}{c}\text { Submodular } \\
\text { mean }(\mathrm{se})\end{array}$ \\
\hline Efficiency & $0.94(0.04)$ & $0.73(0.07)$ & $0.84(0.04)$ & $0.39(0.10)$ \\
& & & & \\
Matching & & & & \\
PAM & $0.94(0.04)$ & $0.01(0.01)$ & $0.84(0.04)$ & $0.08(0.06)$ \\
NAM & $0.00(0.00)$ & $0.73(0.07)$ & $0.00(0.00)$ & $0.39(0.10)$ \\
& & & & \\
Stability & & & & \\
exact & $0.78(0.10)$ & $0.14(0.09)$ & $0.54(0.07)$ & $0.03(0.03)$ \\
-2 tokens & $0.89(0.06)$ & $0.33(0.11)$ & $0.68(0.06)$ & $0.06(0.05)$ \\
-5 tokens & $0.93(0.05)$ & $0.58(0.12)$ & $0.78(0.06)$ & $0.19(0.11)$ \\
\hline \hline
\end{tabular}

Notes: Robust standard errors (reported in parentheses) are obtained by clustering at the session level to account for interdependencies of observations that come from the same session. An efficient outcome is one that maximizes the sum of match surpluses. PAM indicates positive-assortative matching, while NAM indicates negative-assortative matching. An allocation is stable (or stable $-x$ tokens) if the matching is utilitarian efficient and no pair can generate any gain (of more than $\mathrm{x}$ tokens) by blocking the observed outcome.

Table 2 suggests several insights. First, a substantial fraction of all of our markets culminates in efficient matchings. For the most part, matchings are positively assortative when PAM is efficient and negatively assortative when NAM is efficient.

Second, incomplete information poses an important obstacle for efficiency, particularly for submodular markets. For supermodular markets, moving from complete to incomplete information generates a reduction of 10 percentage points in the fraction of efficient matchings. In contrast, for our submodular markets, the fraction of efficient matchings almost halves, dropping by 34 percentage points.

Third, there are fewer stable than efficient outcomes. Much of the instability we observe is due to unstable transfer profiles, as captured by the difference between the fraction of outcomes that 
entail an efficient matching and those that are stable. For example, with complete information, 6\% of supermodular markets do not culminate in an efficient matching, while $16 \%$ of supermodular markets yield an efficient matching but with unstable transfers. This wedge is even more pronounced for submodular markets. With complete information, $27 \%$ of markets do not culminate in efficient matchings, while 59\% produce an efficient matching with unstable transfers.

Fourth, the surplus structure has important impacts on whether emergent outcomes are stable. Submodular markets generate significantly and substantially fewer stable outcomes than do supermodular markets, especially under incomplete information, with differences that are more pronounced than the comparisons of efficient outcomes. With complete information, $78 \%$ of supermodular markets culminate in a stable outcome, while for submodular markets, the corresponding figure is $14 \%$. This is the case even when allowing for minimal "mistakes" in terms of the transfer profile. With incomplete information, the fraction of stable outcomes in supermodular markets falls to $54 \%$, while the fraction of stable outcomes in submodular markets nearly vanishes, standing at $3 \%$.

These comparisons are mirrored by the pattern of blocking pairs. For supermodular markets, unstable outcomes are associated with an average of 1.56 blocking pairs with complete information and an average of 1.65 blocking pairs with incomplete information. The corresponding figures for our submodular markets are 1.67 and 2.86. In particular, with incomplete information, submodular preferences yield significantly more blocking pairs than supermodular preferences (at the 1\% significance level). The patterns are more pronounced when considering efficient, but unstable, outcomes. In those, we see an average of one blocking pair with complete information and an average of 1.25 blocking pairs with incomplete information when surpluses are supermodular. The figures for our submodular markets are 1.32 and 1.93, both significantly higher (at the $1 \%$ significance levels). A similar pattern emerges when considering the average surplus generated by blocking pairs of unstable outcomes, with incomplete information and submodularity leading to greater average surplus available across blocking pairs. ${ }^{14}$ These results suggest that, even with fairly unrestricted and decentralized interactions, market participants facing incomplete information are not gleaning enough information to attain complete-information stability.

\footnotetext{
${ }^{14}$ In supermodular markets that culminated in an unstable outcome, the average surplus available to blocking pairs was 7.06 tokens with complete information and 7.19 tokens with incomplete information. For submodular markets, the corresponding figures were 5.33 and 7.90 tokens. In particular, the greatest number of blocking pairs and available surplus per blocking pair were observed for submodular markets with incomplete information.
} 
Table 3: Players' Final Payoffs

\begin{tabular}{lcccc}
\hline \hline & \multicolumn{2}{c}{ Complete Information } & \multicolumn{2}{c}{ Incomplete Information } \\
& $\begin{array}{c}\text { Supermodular } \\
\text { mean (se) }\end{array}$ & $\begin{array}{c}\text { Submodular } \\
\text { mean (se) }\end{array}$ & $\begin{array}{c}\text { Supermodular } \\
\text { mean (se) }\end{array}$ & $\begin{array}{c}\text { Submodular } \\
\text { mean (se) }\end{array}$ \\
\hline $\begin{array}{l}\text { Payoffs by Type } \\
\text { Low Type }\end{array}$ & $4.11(0.06)$ & $16.07(0.37)$ & $4.41(0.15)$ & $14.72(0.60)$ \\
Medium Type & $15.83(0.12)$ & $23.81(0.25)$ & $15.60(0.51)$ & $24.19(0.14)$ \\
High Type & $35.86(0.10)$ & $38.87(0.45)$ & $35.29(0.27)$ & $36.33(0.54)$ \\
& & & & \\
Equal Splits & & & & \\
All allocations & $0.79(0.04)$ & $0.32(0.01)$ & $0.67(0.06)$ & $0.39(0.06)$ \\
First two deals & $0.64(0.03)$ & $0.60(0.03)$ & $0.15(0.01)$ & $0.23(0.02)$ \\
Efficient allocations & $0.81(0.03)$ & $0.31(0.01)$ & $0.69(0.05)$ & $0.29(0.02)$ \\
Stable allocations & $0.87(0.02)$ & $0.30(0.01)$ & $0.80(0.06)$ & $0.33(0.21)$ \\
\hline \hline
\end{tabular}

Notes: Average payoffs by players' types are reported in the first three rows. Robust standard errors are clustered at the session level and reported in the parentheses. Equal splits correspond to ratios of payoffs between two matched players falling between $45 \%$ and $55 \%$. For the first two deals in the incomplete-information markets, we focus on deals in which the proposer receives non-negative payoffs.

\subsection{Payoffs}

Table 3 provides aggregate statistics of participants' payoffs and transfers. The top panel of the table depicts the average payoffs by type. Theoretically, all stable outcomes of our supermodular markets entail higher types gaining greater payoffs. In our submodular markets, however, a uniform distribution over stable outcomes would yield only $50 \%$ of stable transfer profiles exhibiting this monotonic pattern (see our Appendix B for details on the constraints stability with transfers imposes). Interestingly, when considering average payments, in all of our treatments we see high types garnering significantly greater payoffs than medium types, who end up with significantly greater payoffs than low types. Nonetheless, when we consider these comparisons market by market, we see some differences, echoing our theoretical predictions. When information is complete, all of our supermodular markets, but only $83 \%$ of our submodular markets, end up with higher types gaining greater payoffs. When information is incomplete, $93 \%$ of our supermodular markets and $61 \%$ of our submodular markets generate greater payoffs for higher types.

Equal splits are common in experimental work (e.g. Andreoni and Bernheim, 2009). While equal splits are consistent with stability for supermodular markets, that is not the case for submodular markets. In fact, were participants simply following an egalitarian norm and always proposing an equal split of match surpluses, we would expect to observe PAM outcomes in all our markets, as described in Section 3. Table 2 suggests that egalitarian norms cannot be medi- 
ating all of our results. Indeed, in our submodular markets, we see PAM outcomes quite rarely. In fact, while the transfers required for stable outcomes seem elusive, particularly in submodular markets, the overall efficiency in those markets is far greater than under PAM. While PAM would generate a total surplus of 128 tokens, the top panel of Table 3 indicates an average total surplus of 157.5 tokens under complete information and an average total surplus of 150.5 tokens under incomplete information. In other words, transfers do allow for greater efficiency, even in cases in which stability appears the most challenging to achieve.

The bottom panel of Table 3 displays explicitly the frequency of (roughly) 50 - 50 splits of the match surplus in the final outcomes observed across markets. In order to allow for some margin of error, we consider offers that range from $45 \%$ to $55 \%$ of the surplus. Such roughly equal splits are prevalent, albeit not universal, in our supermodular markets: hovering around $80 \%$ with complete information and around $70 \%$ with incomplete information. They are far less common in our submodular markets, comprising fewer than $40 \%$ of the outcomes regardless of the information available. ${ }^{15}$

Figure 1 describes the cumulative distribution functions corresponding to payoffs across our treatments. While complete-information treatments generally yield more pronounced "jumps" the underlying preferences have a clear impact. With supermodular preferences, many of the payoffs take one of three values. Indeed, when superimposing the distribution of payoffs that would be generated by equally splitting the surplus between agents in the efficient matching (the orange solid line, corresponding to equal masses receiving payoffs of 4,16, and 36), we see substantial overlap. Submodular preferences, however, yield a nearly uniform distribution of payoffs, particularly when information is incomplete. With complete information, we do see a large fraction of individuals receiving a payoff of 24 , an observation we soon elaborate on. Furthermore, the payoff distributions corresponding to submodular preferences first order stochastically dominate those corresponding to supermodular preferences. This, again, suggests that participants are not simply implementing the efficient matching and splitting the surplus in those markets. Indeed, the resulting distribution (depicted in the blue solid line, corresponding to one third of the popu-

\footnotetext{
${ }^{15}$ Interestingly, when looking at initial accepted offers in our markets, we see far more equal shares of match surpluses for matches between congruent types in our submodular markets. In the first two accepted offers, equal sharing occurs in over $89 \%$ of deals between participants of the same type. Nonetheless, even in these additional deals, participants of different types do not split the surpluses equally. In particular, low types matching with high types get only $20 \%$ of the surplus. We provide more details on behavior at the start of our markets' operations in Appendix A.
} 
Figure 1: Cumulative Distributions of Players' Payoffs

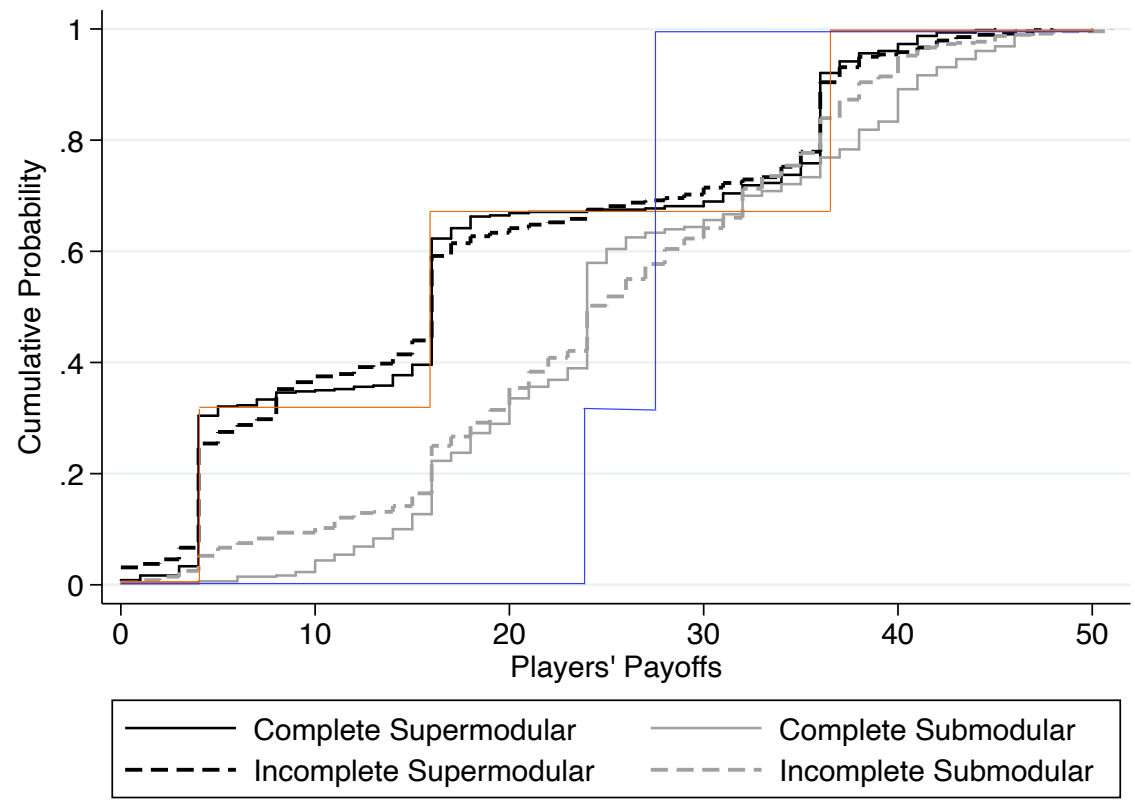

lation receiving a payoff of 24 and two thirds receiving a payoff of 28) would second order, but not first order, stochastically dominate the corresponding distribution for our supermodular markets.

Figure 1 presents payoff distributions aggregated across all market participants. At first blush, it indicates similarity in overall payoff distributions between complete- and incomplete-information markets of the same surplus variety. Figure 2 presents the distribution of payoffs by types of agents in markets that culminated in "nearly stable" outcomes, ones in which the matching is efficient and there is a stable allocation of the surpluses such that each participant's payoff is at most 2 tokens away from what they would receive in that allocation. ${ }^{16}$ The figure depicts how observed payoffs relate to the range of payoffs corresponding to stable outcomes, captured by the red solid line in each panel (see Appendix B for computational details). In line with our discussion above, the distribution of payoffs for all agents and across our markets exhibits a spike at the midpoint of stable allocations. However, the figure conveys several additional messages. Considering our complete-information treatments, payoffs corresponding to the midpoint of the interval of stable transfers are far more prevalent in our supermodular markets. In fact, in our submodular markets, while over $70 \%$ of the medium-type agents receive the mid-point of their stable payoffs, lowand high-type agents have far more disperse payoff distributions. With incomplete information,

\footnotetext{
${ }^{16}$ Allowing for a small margin of error ensures that the samples we discuss are of sufficient size.
} 
Figure 2: Distribution of Payoffs by Types in Stable (-\$2) Allocations
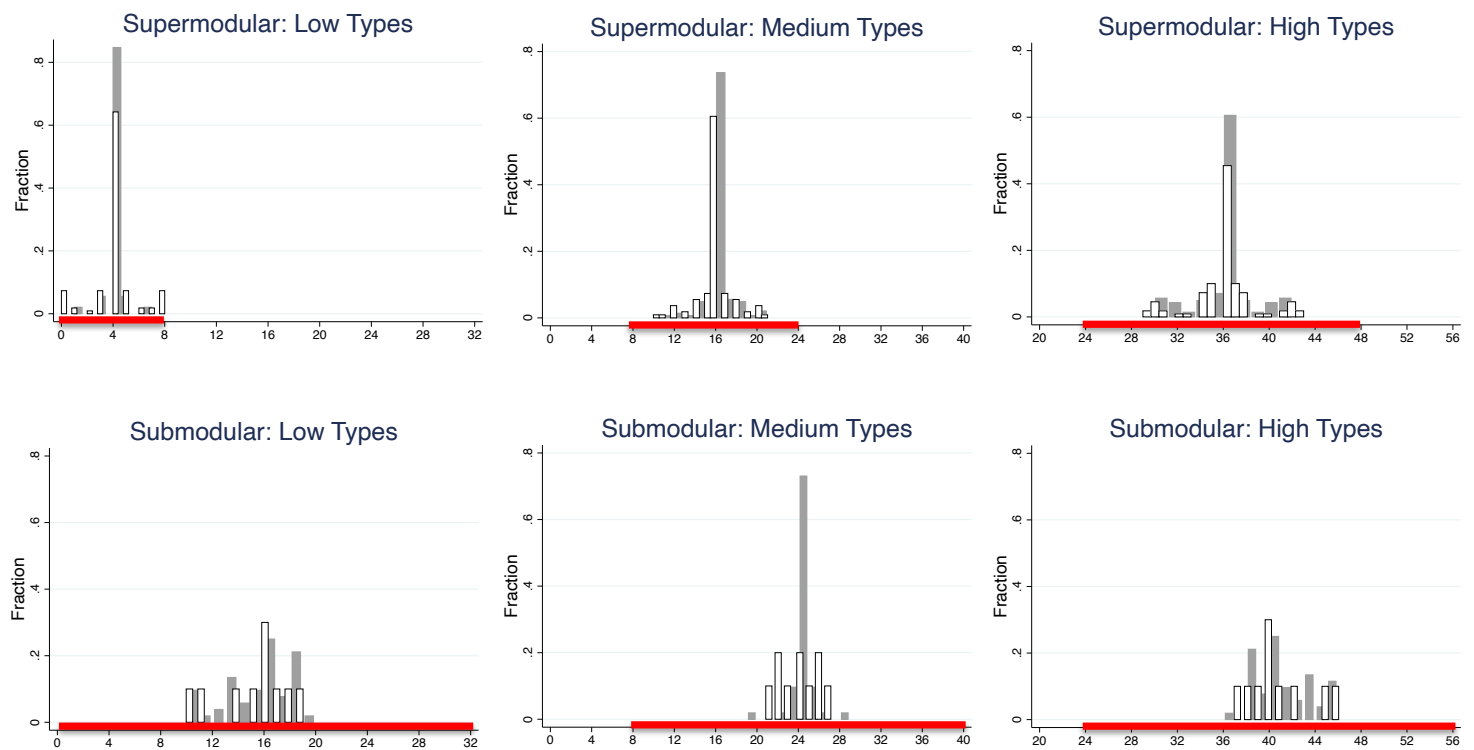

Complete

Incomplete

Notes: The red line on the horizontal axis of each graph depicts the range of payoffs corresponding to stable outcomes predicted by theory.

payoffs are noticeably more diffuse, particularly when preferences are submodular. In fact, for submodular preferences, for all types, no allocation carries more than $30 \%$ of our data.

\subsection{Market Activity}

Table 4 summarizes the aggregate features of activity in our markets. As can be seen, market activity took, on average, between 3 and 5 minutes. Our incomplete-information markets correspond to significantly longer durations and a greater volume of offers made and accepted. Our submodular markets correspond to somewhat longer times to reach an outcome. However, the volume of offers made or accepted does not seem to hinge on the underlying preference structure. At the aggregate level, there are no great differences across agent types in terms of overall offers made or accepted. One exception corresponds to the low-type agents, who make significantly more offers than the high-type agents, particularly when information is incomplete. 
Table 4: Market Characteristics

\begin{tabular}{lcccc}
\hline \hline & \multicolumn{2}{c}{ Complete Information } & \multicolumn{2}{c}{ Incomplete Information } \\
& $\begin{array}{c}\text { Supermodular } \\
\text { mean (se) }\end{array}$ & $\begin{array}{c}\text { Submodular } \\
\text { mean (se) }\end{array}$ & $\begin{array}{c}\text { Supermodular } \\
\text { mean (se) }\end{array}$ & $\begin{array}{c}\text { Submodular } \\
\text { mean (se) }\end{array}$ \\
\hline Market duration (in sec) & $158.6(14.0)$ & $171.5(16.1)$ & $191.6(16.5)$ & $292.9(10.6)$ \\
Nb of offers extended & & & & \\
overall per market & $24.8(1.5)$ & $24.1(3.0)$ & $42.4(4.3)$ & $45.9(5.3)$ \\
by Low type & $9.6(1.4)$ & $10.7(2.0)$ & $19.9(1.5)$ & $21.0(2.7)$ \\
by Medium type & $8.0(0.8)$ & $8.4(0.7)$ & $14.2(2.0)$ & $15.8(2.2)$ \\
by High type & $7.2(0.8)$ & $5.2(0.3)$ & $8.3(0.9)$ & $9.1(1.4)$ \\
& & & & \\
Nb of offers accepted & & & & \\
overall per market & $5.6(0.3)$ & $8.8(1.3)$ & $15.9(1.0)$ & $17.3(1.1)$ \\
by Low type & $1.7(0.04)$ & $2.1(0.26)$ & $5.9(0.36)$ & $5.7(0.38)$ \\
by Medium type & $2.1(0.16)$ & $3.1(0.48)$ & $5.9(0.48)$ & $5.7(0.35)$ \\
by High type & $1.8(0.12)$ & $3.5(0.55)$ & $4.2(0.43)$ & $6.0(0.37)$ \\
\hline \hline
\end{tabular}

Notes: Robust standard errors are clustered at the session level and reported in parentheses.

\subsection{Summary of Aggregate Outcomes}

Overall, participants often establish efficient matchings. Stability is more elusive, indicating that it is easier to settle on an efficient matching than to arrange the attendant transfers. Incomplete information and submodular surplus structures present hurdles to efficiency and especially stability, with the combination of incomplete information and submodularity reducing stability to a tiny percentage of outcomes. Incomplete information appears to introduce "complexity" in that markets with incomplete information take longer to converge and involve substantially more participant interactions to reach an outcome.

\section{Market Dynamics}

\subsection{Match Evolution}

We start by considering the progression of matches over the course of a market's operation. In order to depict our markets' evolution, we inspect quintiles of activity in each market's operation. Namely, for each round in our sessions, we consider the time between the first and last moments of activity. We split that time span into five quintiles, thereby effectively normalizing the length of activity in each of our experimental markets.

Figure 3 reports the mean number of transient and permanent matches formed at each quin- 
Figure 3: Transient and Permanent Matches, by Quintiles

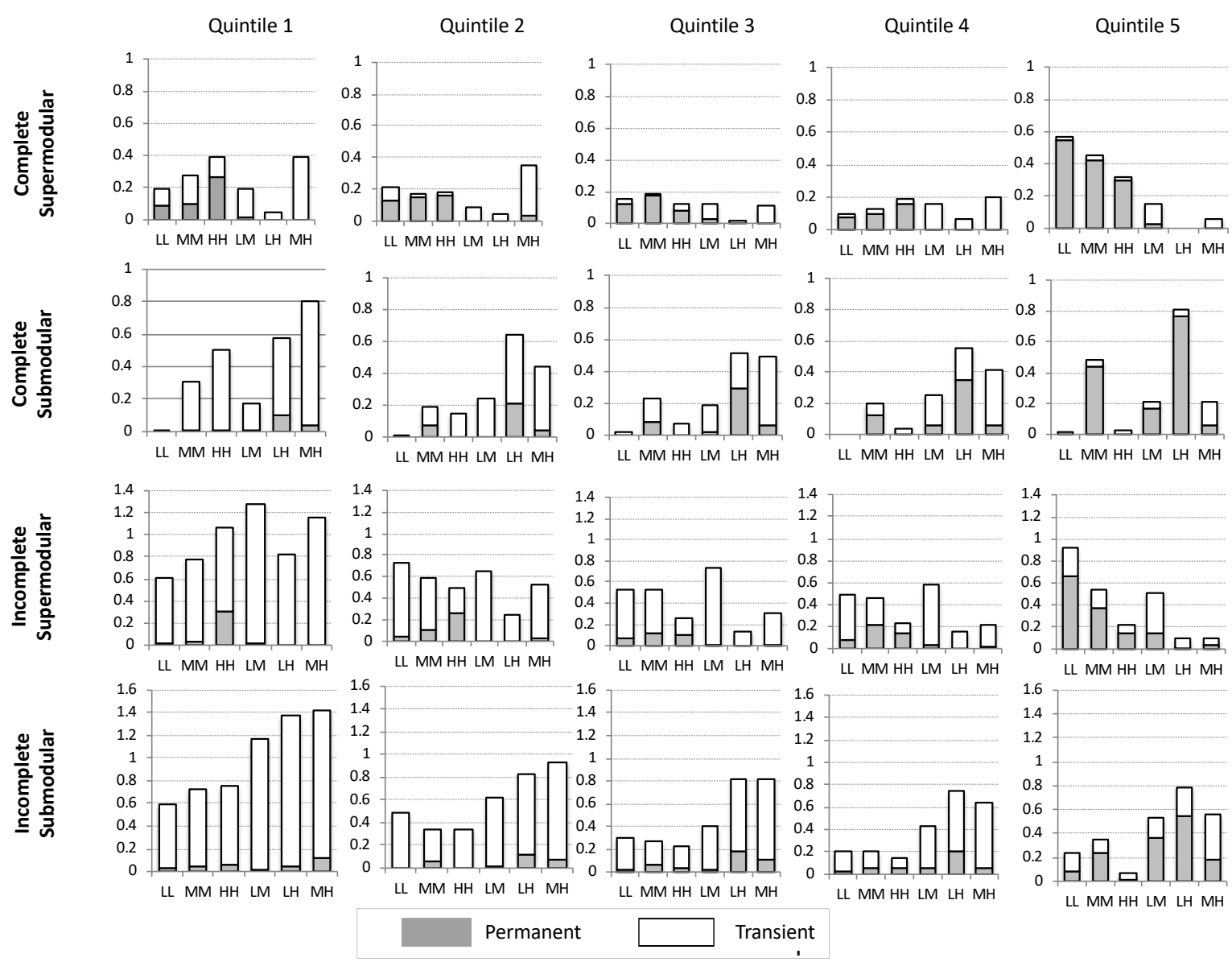

Notes: Quintiles are defined relative to the total time the average market takes to reach a final outcome, where average market duration is calculated as the mean time difference between the last and first activity in that market. The white parts of the bars indicate the transient matches, i.e. those that are broken later on. Grey bars depict the final matches that remain intact until the market's termination.

tile of market duration across treatments, for each combination of matched types-LL denotes two low-type agents, LM denotes one low- and one medium-type agent, and so on. Several observations emerge. First, there are substantial differences in match dynamics across supermodular and submodular markets. The complete-information treatments are particularly telling. With supermodular preferences, both transient and permanent matches are first formed between the high-type agents. They are followed by medium-type agents, and last by low-type agents. In contrast, with submodular preferences, extremal types form matches first: we see the most early transient matches between medium- and high-type agents and the most early permanent matches 
Table 5: Discovering Partners in Incomplete Information Markets

\begin{tabular}{lccccc}
\hline \hline & Quintile 1 & Quintile 2 & Quintile 3 & Quintile 4 & Quintile 5 \\
\hline $\begin{array}{l}\text { Supermodular } \\
\text { all players know partners (frac of markets) }\end{array}$ & 0.14 & 0.38 & 0.51 & 0.56 & 0.60 \\
$\quad$ av \# of players who know their partners & 3.38 & 4.84 & 5.18 & 5.29 & 5.41 \\
& & & & \\
Submodular & & & & \\
$\quad$ all players know partners (frac of markets) & 0.21 & 0.41 & 0.63 & 0.65 & 0.80 \\
$\quad$ av \# of players who know their partners & 3.44 & 4.75 & 5.35 & 5.40 & 5.66 \\
\hline \hline
\end{tabular}

Notes: For each type of market, supermodular or submodular, we report the fraction of markets in which all players discover their partners' surpluses in the corresponding quintile (the first row) and the average number of players (out of six) who discover their partners' surpluses in each quintile (the second row).

between low- and high-type agents. These are followed by matches between congruent mediumtype agents. The early permanent matches between low- and high-type agents occur at substantially lower rates than the corresponding congruent and permanent early matches in our supermodular markets, particularly when accounting for the fact that each market contains two incongruent low- and high-type pairs. Last, we see a substantial fraction of matches between low- and medium-type agents at later market phases. These late matches are not part of stable outcomes.

The second observation from Figure 3 is that incomplete-information treatments are associated with far more offers, in every quintile and particularly early on. Some of these offers act to reveal information about the types of agents on the other side of the market-indeed, early-quintile offers are frequently transient. This presumably explains why some features of the completeinformation markets remain. Specifically, we see more transient and permanent early matches involving high-type agents. In contrast, the lower-type agents take more time to find partners. ${ }^{17}$

\subsection{Learning through Market Operations}

As incomplete-information markets evolve, participants have opportunities to learn others' types. Indeed, whenever an offer is accepted, individuals learn the resulting payoffs and can thus identify the type of individual on the other side of the market. Table 5 documents this type of learning throughout quintiles of our sessions. In both supermodular and submodular markets, by the

\footnotetext{
${ }^{17}$ Repeat offers occur frequently, even in later parts of markets' operations. Consider the average fraction of repeat offers in the second half of each round. In complete-information supermodular markets, these range from 0.27 for low-type agents to 0.34 for medium-type agents to 0.13 for high-type agents. In incomplete-information supermodular markets, these range from 0.34 for low-type agents to 0.29 for medium-type agents to 0.25 for high-type agents. Our submodular markets yield nearly identical frequencies of repeat offers.
} 
third quintile, all participants are fully informed about the surpluses they can generate with all those on the other side of the market in over $50 \%$ of markets, with an average of over 5 out of 6 participants being informed. By the termination of each market, these numbers are even higher. ${ }^{18}$ The experimental protocol also ensures that whenever a match is formed, everyone learns the payoff of the participant accepting the corresponding offer.

Having 5 out of 6 participants informed is sufficient for our stability notion to coincide with complete-information stability, see Section 3. The dearth of stable outcomes in our incompleteinformation treatments is thus puzzling. The following subsection suggests explanations.

\subsection{Patterns of Market Interactions}

Matching theory provides limited guidance on the equilibrating dynamics one might expect in decentralized markets with transfers. We now discuss how certain beliefs might be consistent with a modified version of stability explaining our markets' outcomes. We also illustrate behaviors in our data consistent with participants following a satisficing heuristic, reducing substantially their efforts once hitting a (type-dependent) payoff target.

\subsubsection{Stability with Pessimistic Beliefs}

Suppose that instead of using all the information participants learn from their market interactions, they assemble only the minimal information consistent with the observed final matching. Suppose, further, that participants form pessimistic expectations (from the point of view of blocking), recognizing the possible match surpluses in the market. Namely, when observing a match in which the receiver realizes a payoff of $x$, they assume the matched partner receives the maximal feasible payoff of $72-x$. Then, in those incomplete-information outcomes that fail to be complete-information stable, there is an average of only 0.59 blocking pairs in the supermodular markets and 1.04 blocking pairs in the submodular markets. These numbers are considerably smaller than those reported above for our complete-information markets (1.56 and 1.67, respectively). This indicates that there are beliefs consistent with the information revealed under which participants come as close to stable outcomes as they do under complete information. In prac-

\footnotetext{
${ }^{18}$ Learning in submodular markets appears slightly faster than in our supermodular markets. However, this difference is significant only in the last quintile. Indeed, regression analysis clustering errors at the session level suggests a corresponding $\mathrm{p}$-value of $p=0.026$ when looking at the last quintile, whereas $p>0.10$ for earlier quintiles.
} 
tice, we might expect participants to be less pessimistic and to glean yet more information from the matching process (though perhaps not as much as do the hyper-rational agents in theoretical treatments of incomplete-information stability such as Liu et al., 2014). We conclude that the matching process in incomplete-information markets does not convey enough information to bring the agents to complete-information stability. We leave as an important topic for future work the more refined investigation of whether the resulting behavior reflects stability on the part of timid, under-inferring agents, or failures of less timid and more clever agents to achieve stability.

While pessimistic beliefs provide an effective way to organize the market outcomes we observe, they do not, on their own, explain the dynamics markets follow. ${ }^{19}$ In what follows, we illustrate a simple heuristic consistent with the market activity we observe.

\subsubsection{Satisficing Patterns}

We now consider a satisficing heuristic, whereby participants target a particular payoff, possibly dependent on their market type. Caplin et al. (2011) document the use and prevalence of satisficing heuristics in individual choices. Our design allows for satisficing behavior to emerge in a market setting, where the satisficing threshold depends on global market features. Satisficing would suggest that participants make offers before hitting their target payoff, and cease making offers once that target is achieved.

For each market, we compute the number of offers made by players of each type-low, medium, or high-conditional on their current payoff, excluding unmatched individuals. We then group offers in bins corresponding to participants' current payoff. We normalize the number of offers by the volume of offers made by the corresponding type across all rounds. Table 6 presents the resulting fraction of offers by each type in each of our treatments, conditioning on the current pay bin. Horizontal lines in the table correspond to a satisficing rule, allowing for a $20 \%$ mistake rate. That is, payoffs exceeding those the line represents yield fewer than $20 \%$ of offers. As can be seen, similar qualitative conclusions result from using lower mistake thresholds. ${ }^{20}$

\footnotetext{
${ }^{19}$ For instance, pessimistic beliefs at the start of each market's activity could correspond to each participant suspecting the minimal match surplus available to them. However, initial offers in our incomplete-information markets significantly exceed half that surplus for each of the three types. Furthermore, initial offers do not reflect a persistent fraction other than $50 \%$ of that "pessimistic" surplus.

${ }^{20}$ Similar levels are derived when considering the first and second half of each market's operations separately. In addition, we do not see substantial differences across early and later rounds. Offer rates documented in Table 6 naturally reflect a combination of how active people are conditional on their current pay and how frequently they find themselves with that pay.
} 
Table 6: Frequency of Offers Made Conditional on the Current Pay

\begin{tabular}{|c|c|c|c|c|c|c|}
\hline \multirow[b]{3}{*}{ Current Pay } & \multirow{2}{*}{\multicolumn{2}{|c|}{ Low Type }} & \multicolumn{2}{|c|}{ Supermodular } & \multirow{2}{*}{\multicolumn{2}{|c|}{ High Type }} \\
\hline & & & Medi & m Type & & \\
\hline & Complete & Incomplete & Complete & Incomplete & Complete & Incomplete \\
\hline 1 to 4 & 0.87 & 0.64 & 0.03 & 0.08 & 0.00 & 0.04 \\
\hline 5 to 8 & 0.13 & 0.28 & 0.29 & 0.25 & 0.00 & 0.05 \\
\hline 9 to 12 & 0.00 & 0.05 & 0.28 & 0.23 & 0.03 & 0.16 \\
\hline 13 to 16 & 0.00 & 0.01 & 0.35 & 0.33 & 0.06 & 0.09 \\
\hline 17 to 24 & 0.00 & 0.01 & 0.04 & 0.09 & 0.39 & 0.30 \\
\hline 25 to 32 & 0.00 & 0.00 & 0.01 & 0.01 & 0.33 & 0.23 \\
\hline 33 to 40 & 0.00 & 0.00 & 0.00 & 0.00 & 0.18 & 0.12 \\
\hline \multirow[t]{2}{*}{41 and above } & 0.00 & 0.00 & 0.00 & 0.01 & 0.00 & 0.01 \\
\hline & \multicolumn{6}{|c|}{ Submodular } \\
\hline Current Pay & Complete & Incomplete & Complete & Incomplete & Complete & Incomplete \\
\hline 1 to 4 & 0.33 & 0.26 & 0.00 & 0.06 & 0.00 & 0.00 \\
\hline 5 to 8 & 0.17 & 0.24 & 0.00 & 0.12 & 0.00 & 0.00 \\
\hline 9 to 12 & 0.13 & 0.11 & 0.03 & 0.10 & 0.00 & 0.01 \\
\hline 13 to 16 & 0.27 & 0.24 & 0.27 & 0.17 & 0.01 & 0.01 \\
\hline 17 to 24 & 0.11 & 0.11 & 0.60 & 0.37 & 0.05 & 0.18 \\
\hline 25 to 32 & 0.00 & 0.04 & 0.11 & 0.17 & 0.37 & 0.53 \\
\hline 33 to 40 & 0.00 & 0.00 & 0.00 & 0.01 & 0.51 & 0.23 \\
\hline 41 and above & 0.00 & 0.00 & 0.00 & 0.00 & 0.05 & 0.04 \\
\hline
\end{tabular}

Notes: Fractions represent the frequency of offers of a particular type in a particular market conditional on currentpayoff bin. The horizontal line corresponds to the satisficing thresholds with a $20 \%$ mistake threshold: payoffs beyond the threshold lead to fewer than $20 \%$ of offers.

Estimated satisficing thresholds are remarkably consistent across complete- and incompleteinformation markets across types, with only one exception corresponding to low types in supermodular markets. This consistency is reassuring and suggests that market positions are among the strongest forces shaping our participants' behavior. The next section explores this idea further.

\subsubsection{Bargaining Styles}

We now turn to the various bargaining styles participants used and how they affect outcomes in our markets. The main message is that, while individuals vary widely in how they approach our markets, in terms of the number of offers they make, their insistence on higher shares, etc., their payoffs are not strongly affected by these differing interaction styles. By and large, payoffs are determined by the governing match surpluses: supermodular or submodular, and the position one takes in the market in terms of types.

We focus our discussion on one aspect of behavior: the number of offers made in a market. In the Appendix, we include analogous analyses relating to other aspects: number of matches made throughout the market, the share of surpluses demanded in offers, and which quintile the first 
Table 7: Impact of Number of Offers on Final Payoffs

\begin{tabular}{lccc}
\hline \hline & Low & Medium & High \\
\hline Complete & & & \\
Supermodular & $0.19(0.09)$ & $0.16(0.08)$ & $0.14(0.09)$ \\
Submodular & $0.21(0.07)$ & $0.18(0.06)$ & $0.11(0.11)$ \\
& & & \\
Incomplete & & & \\
Supermodular & $0.25(0.10)$ & $0.17(0.08)$ & $0.10(0.05)$ \\
Submodular & $0.22(0.08)$ & $0.17(0.09)$ & $0.11(0.07)$ \\
\hline \hline
\end{tabular}

(a) Fraction of Offers by Type

\begin{tabular}{lccc|ccc|c}
\hline \hline & $\beta_{\text {low }}$ & $\beta_{\text {med }}$ & $\beta_{\text {high }}$ & $\beta_{\text {low }}=\beta_{\text {med }}$ & $\beta_{\text {low }}=\beta_{\text {high }}$ & $\beta_{\text {med }}=\beta_{\text {high }}$ & $R^{2}(n)$ \\
\hline Complete & -0.17 & -1.77 & 13.33 & 0.35 & 0.12 & 0.11 & $0.94(480)$ \\
Supermodular & $(0.61)$ & $(1.74)$ & $(6.60)$ & & & & \\
Complete & 1.05 & 1.18 & -2.05 & 0.98 & 0.69 & 0.39 & $0.84(480)$ \\
Submodular & $(4.70)$ & $(2.53)$ & $(5.04)$ & & & & \\
Incomplete & -1.44 & $-10.64^{*}$ & -5.72 & 0.04 & 0.45 & 0.44 & $0.93(480)$ \\
Supermodular & $(1.05)$ & $(3.76)$ & $(5.37)$ & & & & \\
Incomplete & -17.72 & $-14.30^{*}$ & -9.96 & 0.79 & 0.41 & 0.63 & $0.74(480)$ \\
Submodular & $(11.11)$ & $(4.81)$ & $(4.43)$ & & & & \\
\hline \hline
\end{tabular}

(b) Payoff Regressions

Notes: Panel (a) reports the average number of offers made by each participant type as a fraction of all offers made in that specific market, with standard deviations in parentheses. Rows add up to 50\%, modulo rounding. Panel (b) reports the estimated coefficients from linear regressions in which the dependent variable is the participants' payoff and the independent variables include the constant, the dummies for medium and high types (leaving the low types as the base group), the round and the round squared, two measures of altruism per participant, two measures of risk attitudes per participant, and the fractions of offers extended by low, medium, and high types. The first three columns in panel (b) report the estimated coefficients, and robust standard errors in parentheses, on the fraction of offers made by low, medium, and high types, respectively. Standard errors are clustered at the session level. The next three columns report $p$-value tests of coefficients based on these regressions. The final column reports the number of observations and the R-squared.

and last matches were created. The messages from these are qualitatively identical.

Panel (a) of Table 7 illustrates, for each of our treatments, the fraction of offers made by type, as well as their standard deviation. ${ }^{21}$ There is substantial variation in the number of offers participants make, reflected in the relatively large standard deviations. While there are some notable differences across types, with lower types generally making more offers than higher types, differences across information or preference treatments are insignificant.

\footnotetext{
${ }^{21}$ Since there are 6 participants in each market, if all made offers at equal volumes, we would expect each to make $16.66 \%$ of the offers observed.
} 
Panel (b) of Table 7 illustrates the impacts of the number of offers on payoffs. In the reported regressions, we explain the final payoff of a participant by their position, market characteristics, and the number of offers they make, allowing for fixed-effects by participant and clustering errors at the session level. The association is insignificant in all but one case, pertaining to medium types in the incomplete-information supermodular markets, where we observe a moderately positive association between fraction of offers made and final payoff.

A similar picture emerges when considering variations at the individual level. We consider participants who are "high earners," receiving a final payoff that is above the average payoff for the role they take in at least 7 of the 10 rounds played. Analogously, we consider "low earners" as those receiving a final payoff below the average for their role in at least 7 of the 10 rounds. "Medium earners" are all those that remain. The distribution of the various types of earners is similar to that which would be generated by random choice, with somewhat more "high earners." 22 We see minute differences between the three categories of earners in terms of fraction of offers made, when controlling for market features, position in the market, session round, and overall number of offers made in the market (see the Appendix for detailed regression results).

Figure 4 summarizes this discussion. For each of our treatments, and each market position, or type, we classify participants according to the fraction of offers they made, divided into 9 categories. We see a substantial number of observations in each of these categories, particularly for those pertaining to fewer than 0.25 of market offers (see Table A.3 in Appendix A). However, as the figure illustrates, we see little impact of this volume of offers on average payoffs for any of the types.

These results are interesting in view of the vast literature suggesting that bargaining strategies may be important in various contexts, most notably in labor markets; see Bertrand (2011) and Shell (2006). While our settings are certainly simplistic in many ways, they suggest a very different story. Market position appears to have a dominant impact on outcomes. Who one matches with, and with what transfer, depends importantly on the role one occupies in the market, while being relatively insensitive to how one bargains.

\footnotetext{
${ }^{22}$ For example, were payoffs determined at random, we would expect $17.19 \%$ of participants to be classified as "high earners." In our data, we see $27.08 \%, 35.42 \%, 31.25 \%$, and $22.92 \%$ high earners in the complete supermodular, incomplete supermodular, complete submodular, and incomplete submodular treatments, respectively.
} 
Figure 4: Payoffs by Type as a Function of Fraction of Offers Made

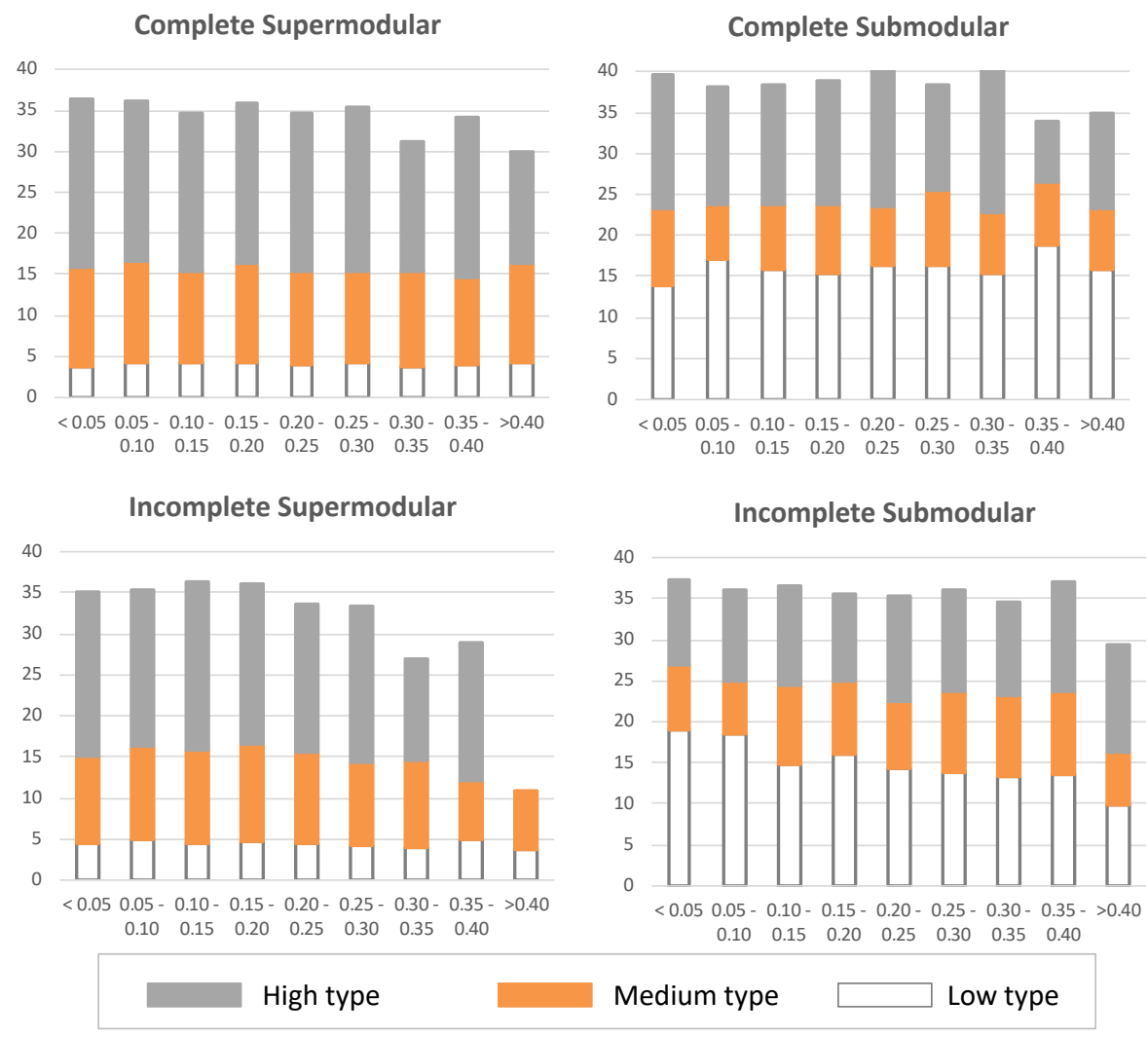

Notes: The height of white bars represents the average payoff of low-type participants in a particular market for a given fraction of market offers they make. Similarly, the heights of the orange and grey bars represent average payoffs of medium- and high-type players, respectively, conditional on the fraction of offers they make in a market.

\section{Conclusions}

We report an array of results examining outcomes and the dynamics of interactions in decentralized matching markets. We allow for transfers and consider markets with complete and incomplete information, and with supermodular or submodular surplus structures.

Without transfers, prior literature has suggested that decentralized interactions often culminate in stable, and hence efficient, outcomes. Our first main message is that, in the presence of transfers, a substantial fraction of outcomes is still efficient, while fewer outcomes are stable. Participants often establish the matches required for efficiency, but find it more difficult to settle on transfers that induce stable outcomes. Our second message is that limited information and the shift from a supermodular to a submodular surplus structure have important impacts. Lim- 
ited information constitutes an important hurdle for stability, even when participants have ample opportunities to learn about others through market interactions. Stability is similarly more challenging to achieve with submodular surpluses. Together, incomplete information and submodularity are deadly for stability-in those markets, less than 5\% of ultimate outcomes are stable. Our third message pertains to the dynamics of interactions. When surpluses are supermodular, high types tend to match first, followed by medium types, and then by low types. When surpluses are submodular, there is substantial variation in the order of match formation and resulting payoffs. Indeed, the ultimate payoff distribution is nearly uniform. Participants' behavior is consistent with a satisficing heuristic in which payoff targets are type dependent. The final message emerging from our data regards bargaining styles. While we see variation in bargaining patterns across individuals, those seem to have little impact on final payoffs. The main determinants of participants' payoffs in our markets are the governing surplus structure and their positions, or types.

We believe our study opens the door for several further investigations, both theoretical and experimental. As described in our literature review, several recent papers have suggested some natural stability notions allowing for incomplete information on one side. The results from those papers cannot be easily reconciled with our data. In part, this may be due to the fact that the suggested notions are agnostic about the decentralized process that takes place in the market. In particular, these solution concepts typically take as fixed a specification of uncertainty and a candidate stable outcome, ignoring the fact that the residual uncertainty is likely to depend on the process by which the candidate outcome is attained. We hope our experimental results might be useful in refining those stability notions to richer settings. Our results point to the need for a model allowing agents to glean complete information about the other side of the market through the matching process, while settling on an outcome that is not complete-information stable. Our experiments also suggest that, when information is incomplete, there might be great value for centralization if (complete-information) stability is an objective. Nonetheless, the extant literature offers little insights on the design of matching markets in the presence of incomplete information, for any objective. We believe this would be a fruitful direction for future research.

Our experiments examine bargaining frictions due to transfers and incomplete information. There are certainly other frictions that would be interesting to inspect. In applications, offers are often costly—in labor markets, employers frequently have limited interview slots or limited offers 
to make, regardless of their yield; in marriage markets, dating is costly in terms of both time and money. Naturally, frictions pertaining to offer costs may dampen market activity. That, in turn, would limit learning even further, as participants "try out" fewer matches. The inefficiencies we identify may then offer lower bounds on the inefficiencies one might expect in practice. There are other details that our basic design does not incorporate: whether offers are exploding, communication channels between market participants, etc. We hope our study sets the stage for further investigations of such additional frictions.

\section{References}

Agranov, M. and M. Elliott (2021): “Commitment and (in)Efficiency: A Bargaining Experiment," Journal of the European Economic Association, 19, 790-838.

Alston, M. (2020): "On the Non-existence of Stable Matches with Incomplete Information," Games and Economic Behavior, 120, 336-344.

Andreoni, J. And B. D. Bernheim (2009): "Social Image and the 50-50 Norm: A Theoretical and Experimental Analysis of Audience Effects," Econometrica, 77, 1607-1636.

Becker, G. S. (1973): “A Theory of Marriage: Part I," Journal of Political Economy, 81, 813-846.

Bertrand, M. (2011): “New Perspectives on Gender," in Handbook of Labor Economics, vol 4b, ed. by O. Ashenfelter and D. Card, Amsterdam: Elsevier, 1543-1590.

Biкhснandani, S. (2017): "Stability with One-sided Incomplete Information," Journal of Economic Theory, 168, 372-399.

Caplin, A., M. Dean, and D. Martin (2011): "Search and Satisficing," American Economic Review, $101,2899-2922$.

Chen, D. L., M. Schonger, And C. Wickens (2016): “oTree-An Open-source Platform for Laboratory, Online, and Field Experiments," Journal of Behavioral and Experimental Finance, 9, 88-97.

Chen, Y. and T. Sönmez (2006): "School Choice: An Experimental Study," Journal of Economic theory, 127, 202-231. 
Chen, Y.-C. And G. Hu (2020): “Learning by Matching,” Theoretical Economics, 15, 29-56.

Echenique, F. And L. Yariv (2013): “An Experimental Study of Decentralized Matching,” .

Ferdowsian, A., M. Niederle, And L. Yariv (2022): “Decentralized Matching with Aligned Preferences," .

Gneezy, U. And J. Potters (1997): “An Experiment on Risk Taking and Evaluation Periods,” The Quarterly Journal of Economics, 112, 631-645.

Hakimov, R. AND D. KüBler (2021): “Experiments on centralized school choice and college admissions: A Survey," Experimental Economics, 24, 434-488.

He, S., J. Wu, and H. Zhang (2022): “Decentralized Matching with Transfers: Experimental and Noncooperative Analyses," .

Kapor, A. J., C. A. Neilson, and S. D. Zimmerman (2020): "Heterogeneous Beliefs and School Choice Mechanisms," American Economic Review, 110, 1274-1315.

Liu, Q. (2022): "Stability and Bayesian Consistency in Two-Sided Markets," American Economic Review, 110, 2625-2666.

Liv, Q., G. J. Mailath, A. Postlewaite, and L. Samuelson (2014): “Stable Matching with Incomplete Information," Econometrica, 82, 541-587.

Nalbantian, H. R. and A. Schotter (1995): "Matching and Efficiency in the Baseball Free-Agent System: An Experimental Examination," Journal of Labor Economics, 13, 1-31.

Niederle, M. and A. E. Roth (2009): “Market Culture: How Rules Governing Exploding Offers Affect Market Performance," American Economic Journal: Microeconomics, 1, 199-219.

PAis, J. ANd Á. Pintér (2008): "School Choice and Information: An Experimental Study on Matching Mechanisms," Games and Economic Behavior, 64, 303-328.

Pais, J., Á. Pintér, And R. F. Veszteg (2020): “Decentralized Matching Markets with(out) Frictions: A Laboratory Experiment," Experimental Economics, 23, 212-239. 
Plott, C., N. Roy, and B. Tong (2013): "Marshall and Walras, Disequilibrium Trades and the Dynamics of Equilibration in the Continuous Double Auction Market," Journal of Economic Behavior and Organization, 94, 109-205.

Roth, A. E. And M. Sotomayor (1990): “Two-Sided Matching: A Study in Game-theoretic Modeling and Analysis," Econometric Society Monographs, 18.

Roth, A. E. And J. H. V. Vate (1990): “Random paths to stability in two-sided matching," Econometrica: Journal of the Econometric Society, 1475-1480.

Shell, G. R. (2006): Bargaining for Advantage: Negotiation Strategies for Reasonable People, Penguin. 


\section{A Additional Analysis}

\section{A.1 Do Markets Start the Same Way?}

Since most of our results consider the entirety of participants' market interactions, one may wonder whether the impact of the surplus variety occurs through market dynamics alone. In this section, we show that even initial activity varies by the sort of surplus that governs preferences. We focus on complete-information markets, since initial interactions in incomplete-information markets are naturally noisy due to the limited knowledge participants have regarding their own and others' preferences.

Figure A.1 illustrates the frequency of matches formed and offers made at the start of our markets. As can be seen, different patterns emerge across our markets. For example, in our supermodular markets, initial matches are mostly formed between stable partners, with a predominance of matches and offers between high types. In contrast, in our submodular markets, while early matches between high types are still prevalent, most offers occur between low and high types, or between medium and high types. Furthermore, there is roughly an equal share of matches between low and high, medium and high, or two medium types.

Table A.2 suggests that the profile of transfers also differs across market classes from the start. Interestingly, submodular markets are characterized by more equal splits at the start of interactions, even though, as we showed in the paper, this pattern reverses as markets evolve.

\begin{tabular}{lcccc}
\multicolumn{2}{c}{ Table A.2: Frequency of Equal Splits in First Deals/Offers } \\
\hline \hline & \multicolumn{2}{c}{ First two deals } & \multicolumn{2}{c}{ First two offers } \\
& Supermodular & Submodular & Supermodular & Submodular \\
\hline LL & 0.78 & & 1.00 & 0.00 \\
LM & 0.71 & 1.00 & 0.64 & 0.50 \\
LH & 0.29 & 0.20 & 0.55 & 0.42 \\
MM & 0.83 & 0.95 & 0.77 & 0.75 \\
MH & 0.47 & 0.60 & 0.59 & 0.70 \\
HH & 0.75 & 0.89 & 0.67 & 0.79 \\
\hline \hline
\end{tabular}

Notes: Equal split corresponds to between $45 \%$ and $55 \%$ of the total surplus.

\section{A.2 Bargaining Styles}

Table A. 3 below is the companion table for Figure 4 in the main text of the paper. It depicts the number of observations we have for each of the categories representing the fraction of offers made 
Figure A.1: First Two Offers and Deals with Complete Information

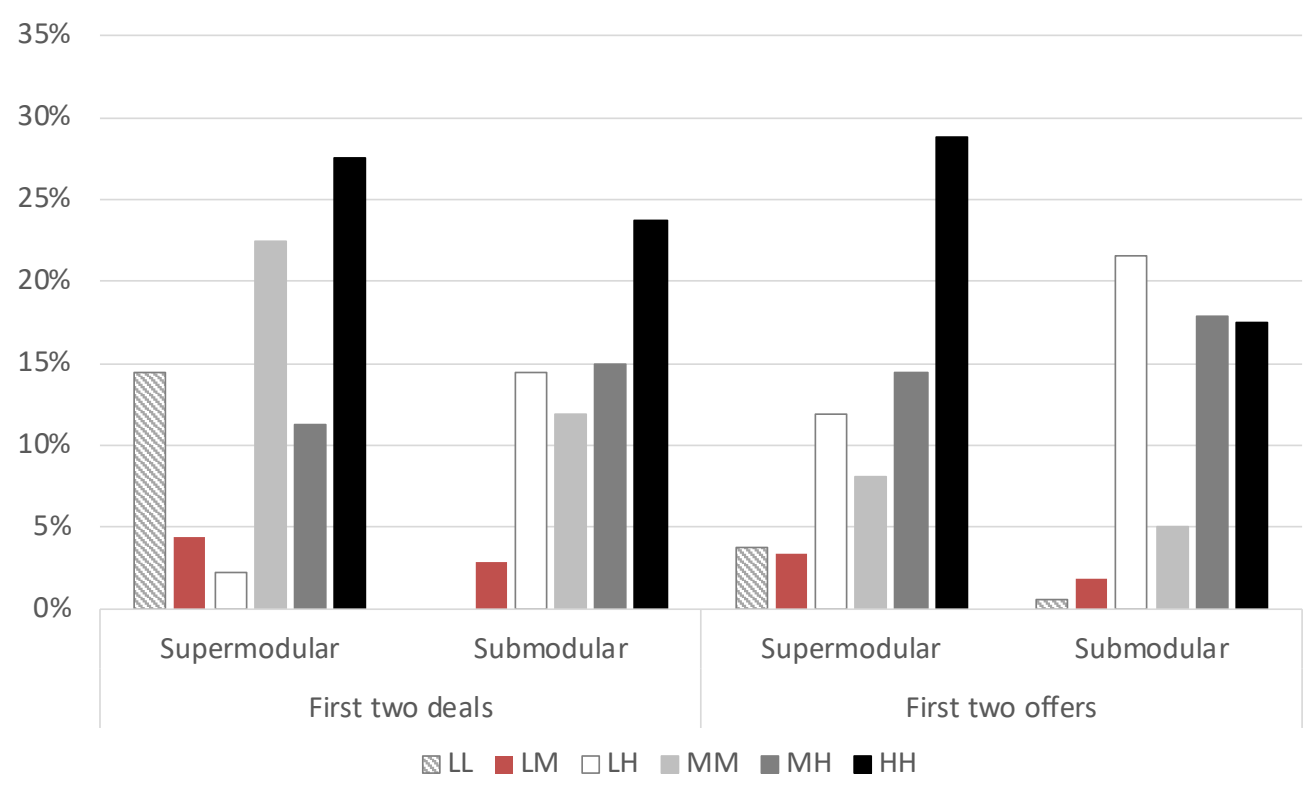

Notes: The frequencies for non-congruent matches (LM, LH, MH) are divided by two, since there are twice as many opportunities to make such offers/deals than congruent ones (LL, MM, and HH).

in a market by a particular player type in a particular market variety. There are 160 observations for each player type in each market ( 8 groups $\times 10$ markets $\times 2$ players of each type).

Next, we replicate the analysis presented in the main body of the paper regarding bargaining styles of market participants with respect to other characteristics of market behavior such as number of matches made throughout the market, the share of surpluses demanded when making offers, and the timing in which the first and last matches were created. For each of these four characteristics, we present the variation across different types of players and the effects of this variation on final payoffs (using regression analysis similar to that reported in Table 6).

In Table A.6 we report tests pertaining to comparisons of coefficients based on regression analysis of the bargaining style of "high earners." Specifically, for each market structure, corresponding to the governing surplus and information available, we run a linear regression in which the dependent variable is one of the market behavior characteristics-number of offers, number of deals, share of surplus requested in offers, and timing of the first and last match-on a constant, indicators for medium- and high-type participants, interaction terms corresponding to high earnings and type, and control variables. The controls include the round number, the fixed effects 
Table A.3: Number of observations for each category in Figure 4

\begin{tabular}{lcccccc}
\hline \hline & \multicolumn{3}{c}{ Complete Supermodular } & \multicolumn{3}{c}{ Complete Submodular } \\
& low & medium & high & low & medium & high \\
\hline less than 0.05 & 21 & 28 & 39 & 6 & 13 & 41 \\
$0.05-0.10$ & 30 & 33 & 26 & 15 & 20 & 45 \\
$0.10-0.15$ & 21 & 23 & 28 & 35 & 33 & 31 \\
$0.15-0.20$ & 19 & 24 & 22 & 23 & 25 & 15 \\
$0.20-0.25$ & 18 & 11 & 19 & 19 & 33 & 12 \\
$0.25-0.30$ & 15 & 16 & 8 & 30 & 12 & 9 \\
$0.30-0.35$ & 16 & 12 & 7 & 14 & 12 & 4 \\
$0.35-0.40$ & 7 & 6 & 5 & 7 & 7 & 1 \\
more than 0.40 & 13 & 7 & 6 & 11 & 5 & 2 \\
& & & & & & \\
\hline & Incomplete Supermodular & Incomplete Submodular \\
& low & medium & high & low & medium & high \\
\hline less than 0.05 & 4 & 14 & 46 & 2 & 17 & 51 \\
$0.05-0.10$ & 9 & 30 & 50 & 15 & 33 & 47 \\
$0.10-0.15$ & 23 & 37 & 33 & 32 & 30 & 22 \\
$0.15-0.20$ & 24 & 23 & 19 & 23 & 21 & 18 \\
$0.20-0.25$ & 30 & 22 & 2 & 26 & 23 & 9 \\
$0.25-0.30$ & 26 & 15 & 17 & 26 & 19 & 8 \\
$0.30-0.35$ & 23 & 6 & 2 & 15 & 7 & 2 \\
$0.35-0.40$ & 7 & 6 & 1 & 8 & 5 & 1 \\
more than 0.40 & 14 & 7 & 0 & 13 & 5 & 2 \\
\hline \hline
\end{tabular}

Notes: We present the number of observations per category; there are 160 observations per market and type.

for individual participants, the total number of offers made in the market in the first set of regressions, and the total number of deals made in the market in the second set of regressions. We cluster standard errors at the session level to account for interdependencies of observations that come from the same session. We report the estimated coefficients and the robust standard errors in parentheses for the interaction terms of high earners with their type and test whether those interaction coefficients are significantly different from zero. If they are, this means that high earners of the specified type behave in a systematically different way than low earners. 
Table A.4: Variation in Market Behavior

\begin{tabular}{l|ccc|ccc}
\hline \hline & \multicolumn{2}{|c|}{ Number of Matches by Type (frac) } & \multicolumn{3}{c}{ Share of Surplus in Offers (out of 100\%) } \\
\hline & Low & Medium & High & Low & Medium & High \\
\hline Complete & & & & & & \\
Supermodular & $0.31(0.06)$ & $0.36(0.06)$ & $0.33(0.06)$ & $49.15(6.58)$ & $49.31(6.36)$ & $55.20(4.79)$ \\
Submodular & $0.24(0.06)$ & $0.34(0.06)$ & $0.40(0.08)$ & $40.53(6.56)$ & $48.85(4.22)$ & $58.67(8.07)$ \\
& & & & & & \\
Incomplete & & & & & & \\
Supermodular & $0.38(0.08)$ & $0.37(0.06)$ & $0.25(0.08)$ & $47.45(7.31)$ & $49.68(7.00)$ & $52.63(7.86)$ \\
Submodular & $0.33(0.06)$ & $0.32(0.07)$ & $0.34(0.09)$ & $43.59(7.49)$ & $50.85(6.69)$ & $55.36(5.95)$ \\
\hline \hline
\end{tabular}

\begin{tabular}{l|ccc|ccc}
\hline \hline & \multicolumn{3}{|c|}{ First Match (quintile) } & \multicolumn{3}{c}{ Last Match (quintile) } \\
\hline Complete & Low & Medium & High & Low & Medium & High \\
Supermodular & $3.02(1.09)$ & $1.98(0.71)$ & $1.88(0.77)$ & $4.00(0.89)$ & $3.58(0.98)$ & $3.15(1.02)$ \\
Submodular & $2.40(0.99)$ & $1.82(0.77)$ & $1.39(0.48)$ & $3.93(0.86)$ & $4.06(0.73)$ & $3.72(0.70)$ \\
& \multicolumn{7}{c}{} & & & & & \\
Incomplete & & & & & & \\
Supermodular & $1.09(0.15)$ & $1.08(0.14)$ & $1.05(0.11)$ & $4.47(0.70)$ & $4.00(0.91)$ & $2.57(0.88)$ \\
Submodular & $1.13(0.24)$ & $1.08(0.19)$ & $1.05(0.12)$ & $4.24(0.70)$ & $3.95(0.85)$ & $3.65(0.97)$ \\
\hline \hline
\end{tabular}


Table A.5: Does Market Behavior Affects Final Payoffs? Regression Analysis

\begin{tabular}{l|ccc|ccc|c}
\hline \hline & $\beta_{\text {low }}$ & $\beta_{\text {med }}$ & $\beta_{\text {high }}$ & $\beta_{\text {low }}=\beta_{\text {med }}$ & $\beta_{\text {low }}=\beta_{\text {high }}$ & $\beta_{\text {med }}=\beta_{\text {high }}$ & $R^{2}(n)$ \\
\hline Complete & & & & & & \\
Supermodular & $0.82(1.22)$ & $-2.53(1.31)$ & $20.10(17.51)$ & 0.18 & 0.32 & 0.30 & $0.94(480)$ \\
Submodular & $-3.67(3.04)$ & $-6.04^{*}(2.44)$ & $2.70(1.79)$ & 0.35 & 0.26 & 0.08 & $0.84(480)$ \\
& & & & & & & \\
Incomplete & & & & & & \\
Supermodular & $2.20(2.19)$ & $-9.13^{*}(3.28)$ & $-9.30^{* *}(2.34)$ & 0.08 & $\mathbf{0 . 0 3}$ & 0.97 & $0.91(480)$ \\
Submodular & $-11.63(6.59)$ & $-8.66^{* *}(2.12)$ & $1.44(5.22)$ & 0.73 & $\mathbf{0 . 0 1}$ & 0.23 & $0.68(480)$ \\
\hline \hline
\end{tabular}

(a) Number of Matches by Type

\begin{tabular}{l|ccc|ccc|c}
\hline \hline & $\beta_{\text {low }}$ & $\beta_{\text {med }}$ & $\beta_{\text {high }}$ & $\beta_{\text {low }}=\beta_{\text {med }}$ & $\beta_{\text {low }}=\beta_{\text {high }}$ & $\beta_{\text {med }}=\beta_{\text {high }}$ & $R^{2}(n)$ \\
\hline Complete & & & & & & & \\
Supermodular & $0.01(0.01)$ & $0.01(0.02)$ & $0.02(0.02)$ & 0.83 & 0.57 & 0.61 & $0.92(428)$ \\
Submodular & $-0.02(0.07)$ & $-0.04(0.02)$ & $-0.01(0.05)$ & 0.81 & 0.81 & 0.37 & $0.83(448)$ \\
& & & & & & & \\
Incomplete & & & & & & & \\
Supermodular & $0.003(0.01)$ & $0.10(0.05)$ & $0.04(0.04)$ & 0.19 & 0.48 & 0.22 & $0.91(397)$ \\
Submodular & $0.05(0.04)$ & $-0.04(0.09)$ & $0.14(0.08)$ & 0.46 & 0.52 & $\mathbf{0 . 0 5}$ & $0.69(427)$ \\
\hline \hline
\end{tabular}

(b) Share of Surplus in Offers

\begin{tabular}{l|ccc|ccc|c}
\hline \hline & $\beta_{\text {low }}$ & $\beta_{\text {med }}$ & $\beta_{\text {high }}$ & $\beta_{\text {low }}=\beta_{\text {med }}$ & $\beta_{\text {low }}=\beta_{\text {high }}$ & $\beta_{\text {med }}=\beta_{\text {high }}$ & $R^{2}(n)$ \\
\hline Complete & & & & & & & \\
Supermodular & $-0.04(0.03)$ & $0.17(0.11)$ & $-0.03(0.08)$ & 0.23 & 0.86 & 0.14 & $0.96(478)$ \\
Submodular & $-0.29(0.15)$ & $0.21(0.14)$ & $0.43(0.51)$ & $\mathbf{0 . 0 2}$ & 0.29 & 0.71 & $0.84(480)$ \\
& & & & & & & \\
Incomplete & & & & & & \\
Supermodular & $-0.38(0.55)$ & $0.84^{* *}(0.16)$ & $0.54^{*}(0.16)$ & 0.12 & 0.23 & 0.26 & $0.91(480)$ \\
Submodular & $-0.26(0.97)$ & $0.99(1.91)$ & $-0.65(1.28)$ & 0.30 & 0.50 & 0.15 & $0.68(480)$ \\
\hline \hline
\end{tabular}

(c) Timing of First Match (quintile)

\begin{tabular}{l|ccc|ccc|c}
\hline \hline & $\beta_{\text {low }}$ & $\beta_{\text {med }}$ & $\beta_{\text {high }}$ & $\beta_{\text {low }}=\beta_{\text {med }}$ & $\beta_{\text {low }}=\beta_{\text {high }}$ & $\beta_{\text {med }}=\beta_{\text {high }}$ & $R^{2}(n)$ \\
\hline Complete & & & & & & & \\
Supermodular & $0.006(0.03)$ & $-0.01(0.13)$ & $0.30(0.17)$ & 0.92 & 0.21 & 0.09 & $0.96(466)$ \\
Submodular & $-0.37(0.28)$ & $-0.19(0.23)$ & $0.11(0.36)$ & 0.42 & 0.51 & 0.61 & $0.84(476)$ \\
& & & & & & & \\
Incomplete & & & & & & \\
Supermodular & $0.16(0.17)$ & $-0.26(0.24)$ & $-0.47(0.16)$ & 0.38 & 0.00 & 0.61 & $0.91(480)$ \\
Submodular & $-0.85(0.74)$ & $-0.83(0.30)$ & $0.43(0.28)$ & 0.98 & 0.19 & 0.10 & $0.68(480)$ \\
\hline \hline
\end{tabular}

\section{(d) Timing of Last Match (quintile)}

Notes: Each row corresponds to one regression. The dependent variable is the final payoff of a player. The independent variables contain a constant, the dummies for medium- and high-type players, interaction between the relevant characteristics of market behavior (number of deals, share of surplus, timing of the first and the last match) and player type, as well as individual controls for risk attitudes and degree of altruism as captured by the auxiliary tasks conducted at the end of the experiment. We cluster the standard errors by session. The number of observations in the panel (b) is smaller than in other panels because some participants never made an offer in a particular market. 
Table A.6: Individual Analysis of Bargaining Styles and Its Effect on Payoffs

\begin{tabular}{|c|c|c|c|c|c|c|}
\hline & \multicolumn{3}{|c|}{ Number of Offers } & \multicolumn{3}{|c|}{ Number of Deals } \\
\hline & Low & Medium & High & Low & Medium & High \\
\hline Complete & & & & & & \\
\hline Supermodular & $0.05^{* *}(0.01)$ & $0.003(0.01)$ & $0.0001(0.01)$ & $-0.003(0.02)$ & $-0.02^{* *}(0.006)$ & $-0.01(0.02)$ \\
\hline Submodular & $0.07^{*}(0.03)$ & $0.02^{* *}(0.006)$ & $0.02(0.02)$ & $0.02(0.02)$ & $0.01(0.02)$ & $0.03(0.02)$ \\
\hline Incomplete & & & & & & \\
\hline Supermodular & $0.11^{* *}(0.02)$ & $0.10^{* *}(0.01)$ & $0.11^{* *}(0.01)$ & $-0.10^{* *}(0.03)$ & $-0.08^{* *}(0.009)$ & $-0.04(0.02)$ \\
\hline \multirow[t]{3}{*}{ Submodular } & $0.18^{* *}(0.02)$ & $0.14^{* *}(0.03)$ & $0.19^{* *}(0.03)$ & $0.01(0.03)$ & $-0.02(0.04)$ & $-0.00004(0.0002)$ \\
\hline & \multicolumn{3}{|c|}{ Share of Surplus in Offers } & & & \\
\hline & Low & Medium & High & & & \\
\hline Complete & & & & & & \\
\hline Supermodular & $3.77^{* *}(0.66)$ & $4.47^{* *}(1.18)$ & $1.97^{*}(0.75)$ & & & \\
\hline Submodular & $3.84(2.45)$ & $5.74^{* *}(1.57)$ & $5.11^{* *}(1.17)$ & & & \\
\hline Incomplete & & & & & & \\
\hline Supermodular & $1.29(1.97)$ & $1.31(0.61)$ & $0.48(1.32)$ & & & \\
\hline \multirow[t]{3}{*}{ Submodular } & $-7.17^{* *}(1.64)$ & $-7.80^{* *}(1.89)$ & $-3.22^{* *}(0.76)$ & & & \\
\hline & \multicolumn{3}{|c|}{ Timing of First Deal (in quintiles) } & \multicolumn{3}{|c|}{ Timing of Last Deal (in quintiles) } \\
\hline & Low & Medium & High & Low & Medium & High \\
\hline Complete & & & & & & \\
\hline Supermodular & $0.31(0.25)$ & $-0.09(0.11)$ & $-0.30(0.22)$ & $0.58^{* *}(0.12)$ & $-0.12(0.09)$ & $-0.08(0.12)$ \\
\hline $\begin{array}{l}\text { Submodular } \\
\text { Incomplete }\end{array}$ & $-1.34^{* *}(0.12)$ & $-1.28^{* *}(0.20)$ & $-1.37^{* *}(0.18)$ & $-0.04(0.06)$ & $-0.39(0.18)$ & $-0.20(0.14)$ \\
\hline Supermodular & $-0.11(0.06)$ & $-0.10^{* *}(0.01)$ & $-0.09^{*}(0.04)$ & $-0.48(0.36)$ & $-0.69^{* *}(0.16)$ & $-0.19(0.11)$ \\
\hline Submodular & $0.05(0.03)$ & $0.12^{* *}(0.02)$ & $0.11^{* *}(0.02)$ & $0.42(0.34)$ & $0.27(0.20)$ & $1.00^{* *}(0.16)$ \\
\hline
\end{tabular}

Notes: see description above 


\section{B Predictions from Stability with Transfers}

As described in Section 3, a stable outcome is an individually rational outcome that has no blocking pairs. When transfers are available, there exist many transfer profiles that sustain stable outcomes in both our supermodular and submodular markets. The associated stable matching, however, is unique for both market types. The table below shows the system of inequalities for each market type that identifies the set of stable transfer profiles. We use $x_{i}$ to denote the payoff of market participant $i$, using the labels in Table 1 in the main text. The first column corresponds to our supermodular market, the second to our submodular market.

\begin{tabular}{l|l}
\hline Supermodular market & Submodular market \\
\hline$x_{\text {Kiwi }} \geq 0$ & $x_{\text {Kiwi }} \geq 0$ \\
$x_{\text {Mango }} \geq 0$ & $x_{\text {Mango }} \geq 0$ \\
$x_{\text {Plum }} \geq 0$ & $x_{\text {Plum }} \geq 0$ \\
$x_{\text {Blue }} \geq 0$ & $x_{\text {Blue }} \geq 0$ \\
$x_{\text {Crimson }} \geq 0$ & $x_{\text {Crimson }} \geq 0$ \\
$x_{\text {White }} \geq 0$ & $x_{\text {White }} \geq 0$ \\
$x_{\text {Kiwi }}+x_{\text {Blue }} \geq 8$ & $x_{\text {Kiwi }}+x_{\text {Blue }} \geq 8$ \\
$x_{\text {Kiwi }}+x_{\text {Crimson }} \geq 16$ & $x_{\text {Kiwi }}+x_{\text {Crimson }} \geq 32$ \\
$x_{\text {Kiwi }}+x_{\text {White }} \geq 24$ & $x_{\text {Kiwi }}+x_{\text {White }} \geq 56$ \\
$x_{\text {Mango }}+x_{\text {Blue }} \geq 16$ & $x_{\text {Mango }}+x_{\text {Blue }} \geq 32$ \\
$x_{\text {Mango }}+x_{\text {Crimson }} \geq 32$ & $x_{\text {Mango }}+x_{\text {Crimson }} \geq 48$ \\
$x_{\text {Mango }}+x_{\text {White }} \geq 48$ & $x_{\text {Mango }}+x_{\text {White }} \geq 64$ \\
$x_{\text {Plum }}+x_{\text {Blue }} \geq 24$ & $x_{\text {Plum }}+x_{\text {Blue }} \geq 56$ \\
$x_{\text {Plum }}+x_{\text {Crimson }} \geq 48$ & $x_{\text {Plum }}+x_{\text {Crimson }} \geq 64$ \\
$x_{\text {Plum }}+x_{\text {White }} \geq 72$ & $x_{\text {Plum }}+x_{\text {White }} \geq 72$ \\
$x_{\text {Kiwi }}+x_{\text {Mango }}+x_{\text {Plum }}+$ & $x_{\text {Kiwi }}+x_{\text {Mango }}+x_{\text {Plum }}+$ \\
$\quad+x_{\text {Blue }}+x_{\text {Crimson }}+x_{\text {White }} \geq 112$ & $+x_{\text {Blue }}+x_{\text {Crimson }}+x_{\text {White }} \geq 160$ \\
\hline
\end{tabular}




\section{Sample Instructions}

Welcome. Welcome to EBEL and thank you for participating in today's experiment. Please place all of your personal belongings away so that we can have your complete attention. Please use the laptops as instructed. In particular, please do not attempt to browse the web or use programs unrelated to the experiment.

Guidelines. You will be paid in private and in cash at the end of the experiment. The amount that you ultimately earn in the experiment depends on your decisions, the decisions of others, and random chance. You have each earned a \$10 payment for showing up on time. You will be using laptops for the entire experiment, and all interactions between yourself and others will take place via the laptop?s terminal. Please DO NOT socialize or talk.

Overview. Today's experiment is about matching. The main part of the experiment consists of 11 rounds: one practice round and 10 actual rounds. We will also ask you to complete several simple tasks at the end.

At the beginning of the experiment, you will be randomly assigned a role: either a color or a food. Your role will remain fixed across all rounds of the experiment. At the beginning of each round, you will be randomly assigned a type:

- If you are a color, you can be one of three types: Blue, Crimson, or White.

- If you are a food, you can be one of three types: Kiwi, Mango, or Plum.

Your type (but not your role) may change across each round of the experiment.

In each round, you will be randomly assigned to a group of six: three colors (one Blue, one Crimson, and one White) and three foods (one Kiwi, one Mango, and one Plum). Your goal in each round is to match with an individual from the opposite role. If you are a color, your goal is to match with a food. If you are a food, your goal is to match with a color.

\section{Match Payoff.}

\begin{tabular}{c|c|c|c}
\hline & KIWI & MANGO & PLUM \\
\hline BLUE & 8 & 16 & 24 \\
\hline CRIMSON & 16 & 32 & 48 \\
\hline WHITE & 24 & 48 & 72 \\
\hline
\end{tabular}


A color and food who match together earn a match payoff (in tokens). You will earn a different payoff depending on whom you match with and how you choose to split the match payoff.

Example 1:

\begin{tabular}{c|c|c|c}
\hline & KIWI & MANGO & PLUM \\
\hline BLUE & 8 & $\mathbf{1 6}$ & 24 \\
\hline CRIMSON & 16 & 32 & 48 \\
\hline WHITE & 24 & 48 & 72 \\
\hline
\end{tabular}

Blue and Mango can match together, with the sum of payoffs for Blue and Mango yielding 16 tokens. For example, Blue could receive a payoff of 10 tokens and Mango would then receive a payoff of 6 tokens. Another example: Blue could receive a payoff of 7 tokens and Mango would then receive a payoff of 9 tokens.

Example 2:

\begin{tabular}{c|c|c|c}
\hline & KIWI & MANGO & PLUM \\
\hline BLUE & 8 & 16 & 24 \\
\hline CRIMSON & 16 & 32 & 48 \\
\hline WHITE & 24 & 48 & 72 \\
\hline
\end{tabular}

Crimson and Plum can match together, with the sum of payoffs for Crimson and Plum yielding 48 tokens. For example, Crimson could receive a payoff of 30 tokens and Plum would then receive a payoff of 18 tokens. Another example: Crimson could receive a payoff of 23 tokens and Plum would then receive a payoff of 25 tokens.

\section{Example 3:}

\begin{tabular}{c|c|c|c}
\hline & KIWI & MANGO & PLUM \\
\hline BLUE & 8 & 16 & 24 \\
\hline CRIMSON & 16 & 32 & 48 \\
\hline WHITE & 24 & 48 & 72 \\
\hline
\end{tabular}

White and Kiwi can match together, with the sum of payoffs for White and Kiwi yielding 24 tokens. For example, White could receive a payoff of 18 tokens and Kiwi would then receive a payoff of 6 tokens. Another example: White could receive a payoff of 9 tokens and Kiwi would then receive a payoff of 15 tokens. 
Information about the payoff matrix. At the beginning of each round, we will reshuffle the rows and columns of the matrix.

\begin{tabular}{c|c|c|c}
\hline & KIWI & MANGO & PLUM \\
\hline BLUE & 8 & 16 & 24 \\
\hline CRIMSON & 16 & 32 & 48 \\
\hline WHITE & 24 & 48 & 72 \\
\hline
\end{tabular}

\section{Examples:}

\begin{tabular}{c|c|c|c}
\hline & KIWI & MANGO & PLUM \\
\hline BLUE & 8 & 16 & 24 \\
\hline CRIMSON & 16 & 32 & 48 \\
\hline WHITE & 24 & 48 & 72 \\
\hline & \multicolumn{1}{|c|}{ KIWI } & MANGO & PLUM \\
\hline BLUE & 24 & 16 & 8 \\
\hline CRIMSON & 48 & 32 & 16 \\
\hline WHITE & 72 & 48 & 24 \\
\hline
\end{tabular}

\begin{tabular}{c|c|c|c}
\hline & KIWI & MANGO & PLUM \\
\hline BLUE & 16 & 32 & 48 \\
\hline CRIMSON & 24 & 48 & 72 \\
\hline WHITE & 8 & 16 & 24 \\
\hline & \multicolumn{1}{|c|}{ KIWI } & MANGO & PLUM \\
\hline BLUE & 48 & 32 & 16 \\
\hline CRIMSON & 72 & 48 & 24 \\
\hline WHITE & 24 & 16 & 8 \\
\hline
\end{tabular}

All possible reshufflings are equally likely. At the beginning of each round, you will not know how the matrix was reshuffled, but you will know which payoffs you might receive.

Examples. Suppose you are Blue, and you learn that your payoffs might be $16,32,48$. It might be that a match with Kiwi generates 16, a match with Mango generates 32, and a match with Plum generates 48 .

\begin{tabular}{c|c|c|c}
\hline & KIWI & MANGO & PLUM \\
\hline BLUE & 16 & 32 & 48 \\
\hline CRIMSON & 24 & 48 & 72 \\
\hline WHITE & 8 & 16 & 24 \\
\hline
\end{tabular}

It might be that a match with Kiwi generates 48, a match with Mango generates 32, and a match with Plum generates 16.

\begin{tabular}{c|c|c|c}
\hline & KIWI & MANGO & PLUM \\
\hline BLUE & 48 & 32 & 16 \\
\hline CRIMSON & 72 & 48 & 24 \\
\hline WHITE & 24 & 16 & 8 \\
\hline
\end{tabular}

All possible reshufflings are equally likely. While you will know your possible payoffs, you will not know which payoff corresponds to which matching. Your payoffs will appear on the top 
part of your interface. Note that no other subject in your group observes your possible match payoffs. Each person only sees his or her own possible match payoffs.

Rules of the Experiment. In each round, you will start off unmatched. You are free to make at most one match proposal to any individual of the opposite role at any given time.

A match proposal must specify what your partner will get. If your proposal is accepted, then you will get the match payoff minus what your partner will get. You are also free to accept or reject match proposals that you receive from other individuals. Any match proposal that is not accepted or rejected within 10 seconds will automatically disappear. When a match is accepted, both the proposer and the receiver see the payoffs they get from the match.

Example 1: Suppose you are Blue, and you know your possible match payoffs are 16, 32, and 48. If you make an offer of 10 to Kiwi, which gets accepted, you might see that you get 6 and Kiwi gets 10. This implies that you and Kiwi have a match payoff of $6+10=16$.

Example 2: Suppose you are Blue, and you know your possible match payoffs are 16, 32, and 48. If you make an offer of 10 to Kiwi, which gets accepted, you might see that you get 38 and Kiwi gets 10. This implies that you and Kiwi have a match payoff of $38+10=48$.

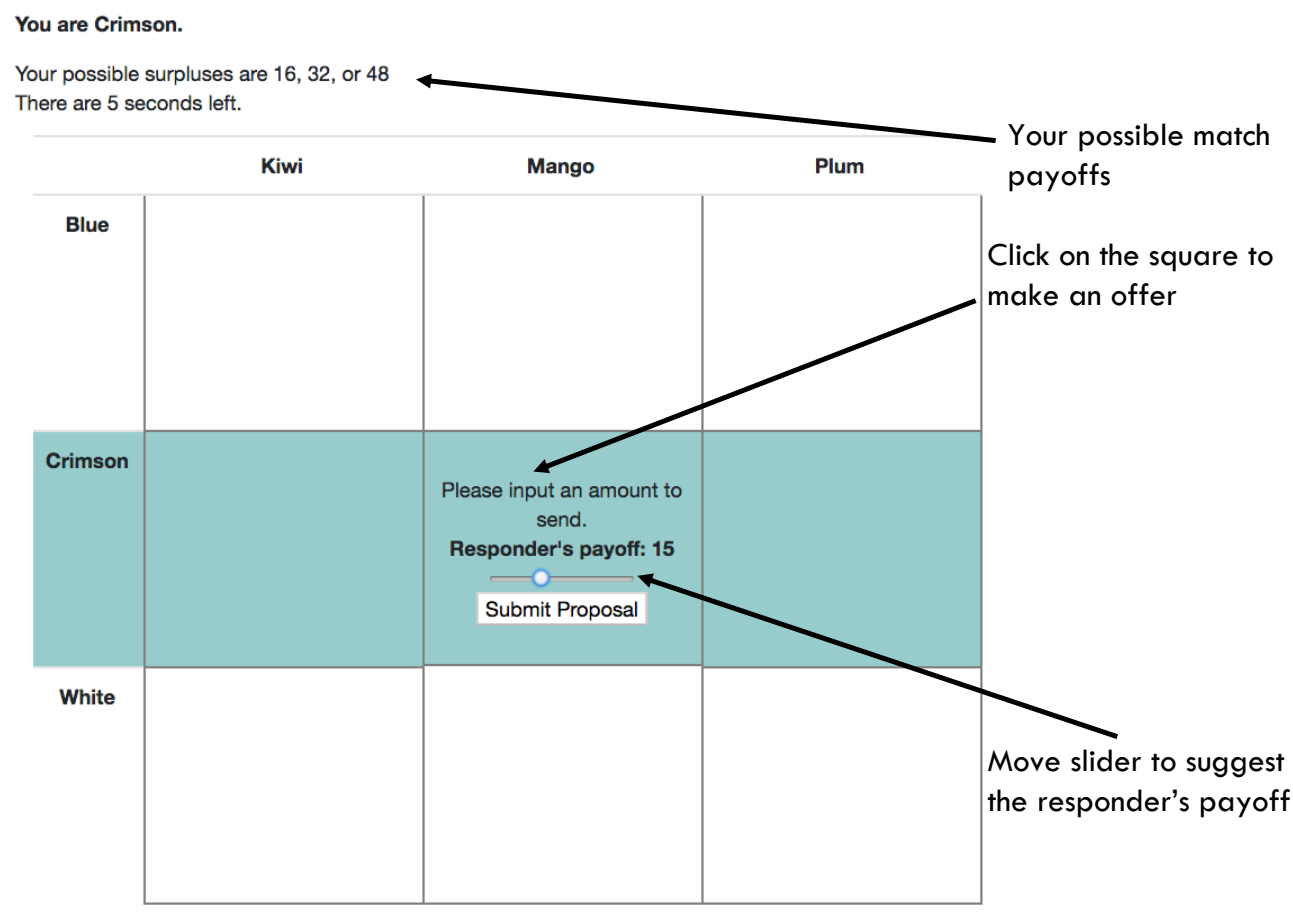


You are Crimson.

Your possible surpluses are 16,32 , or 48

There are 26 seconds left.

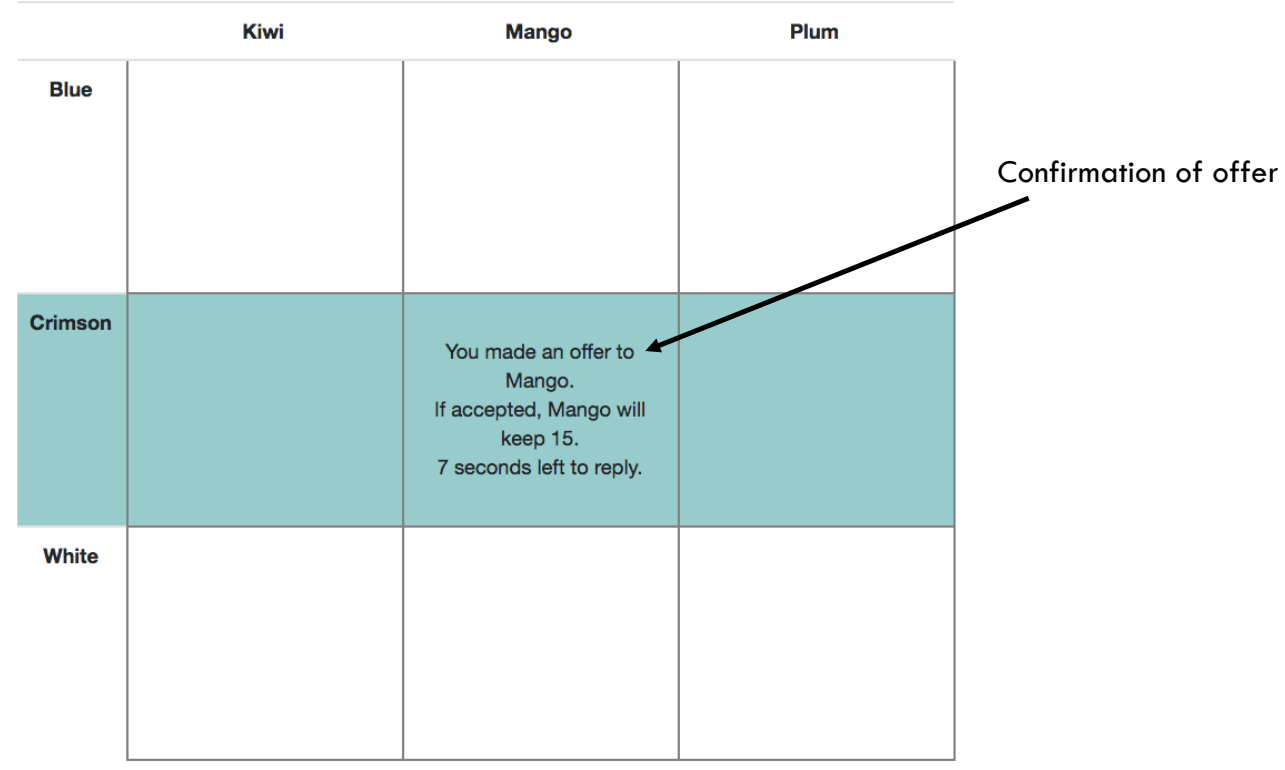


You are Mango.

Your possible surpluses are 8,16 , or 24 There are 20 seconds left.

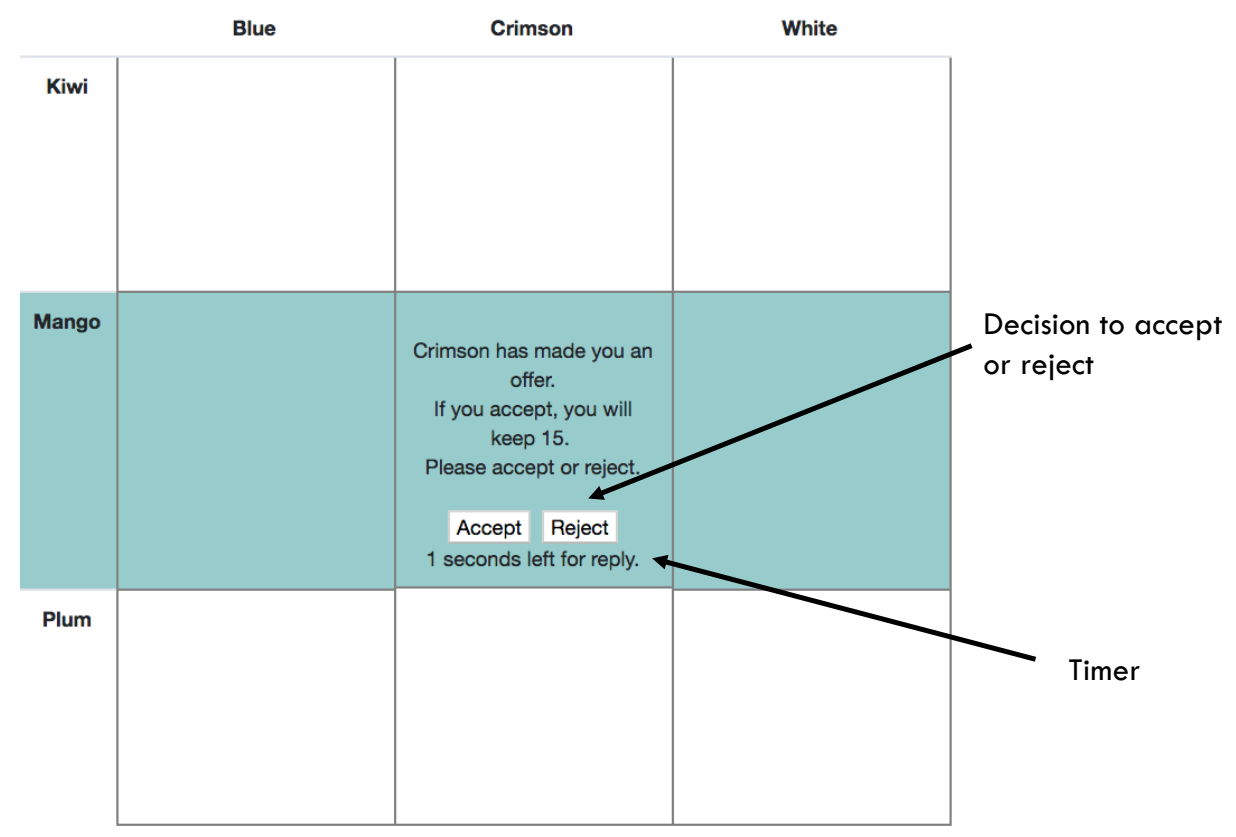




\section{You are Crimson.}

Your possible surpluses are 8,16 , or 24

There are 26 seconds left.

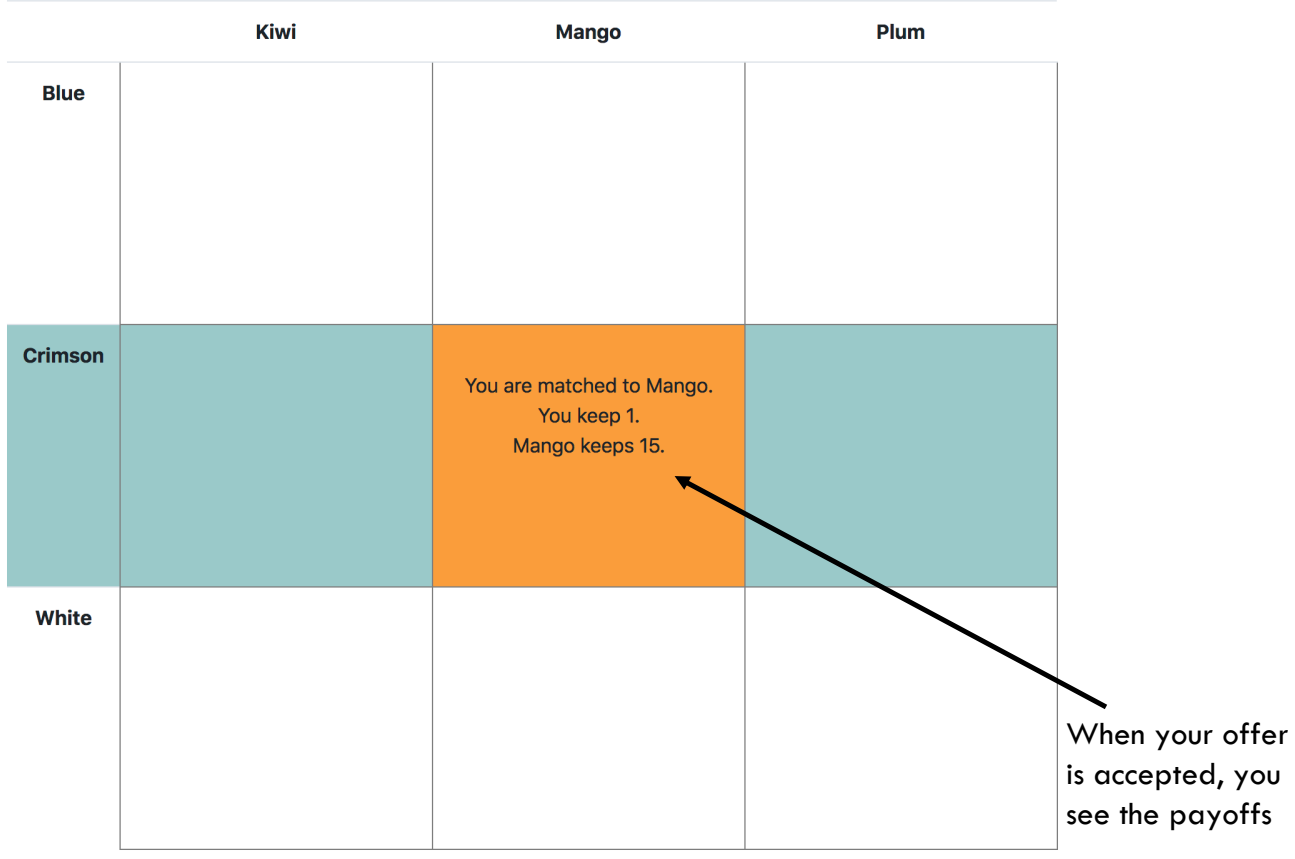




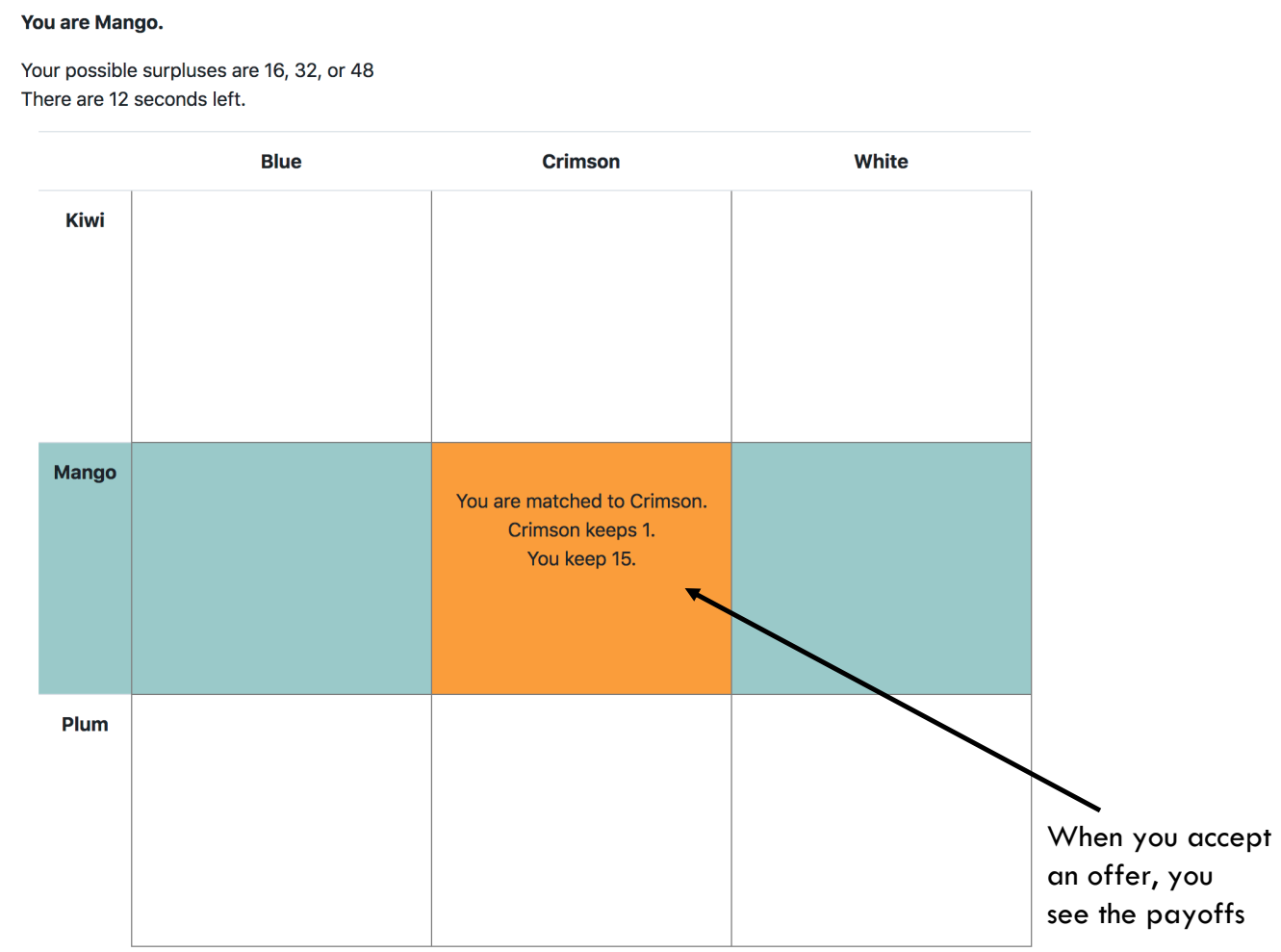


You are Kiwi.

Your possible surpluses are 16,32 , or 48 There are 16 seconds left.

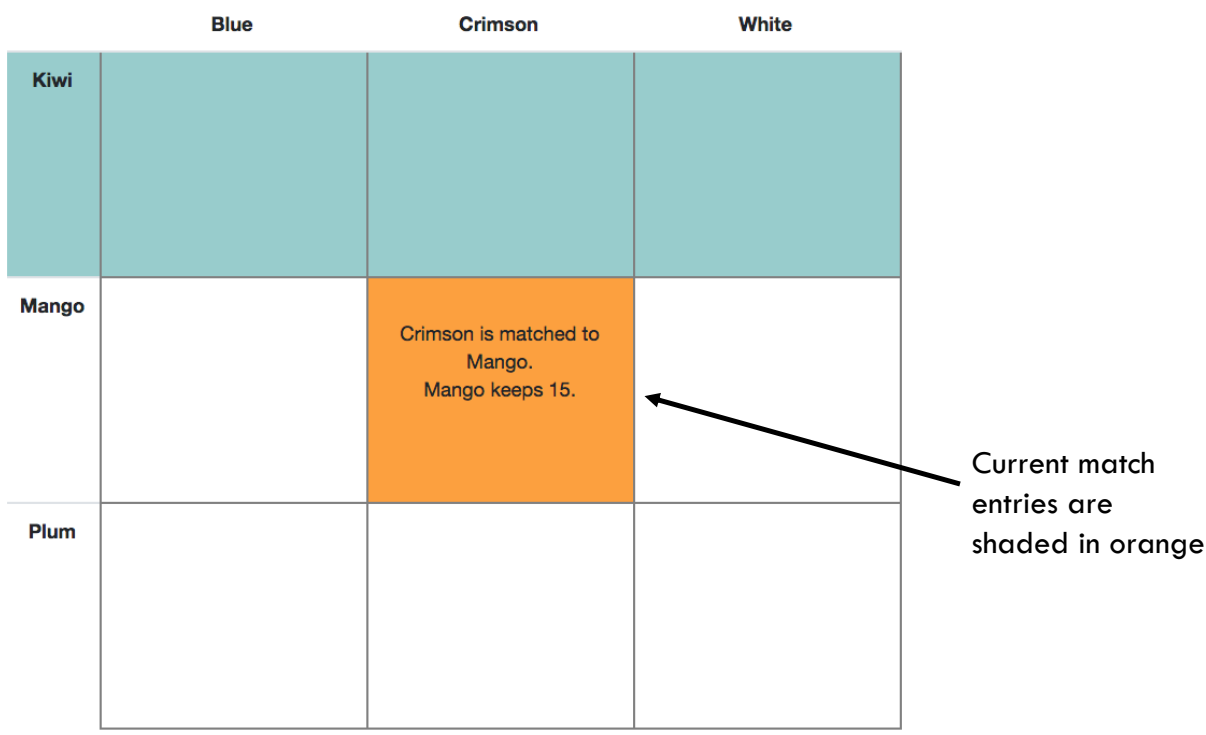


Rules of the Experiment, cont... If you proposed a match which was accepted and in which your own earnings are negative, then you will have an opportunity to unilaterally break this match by clicking a "cancel" button.

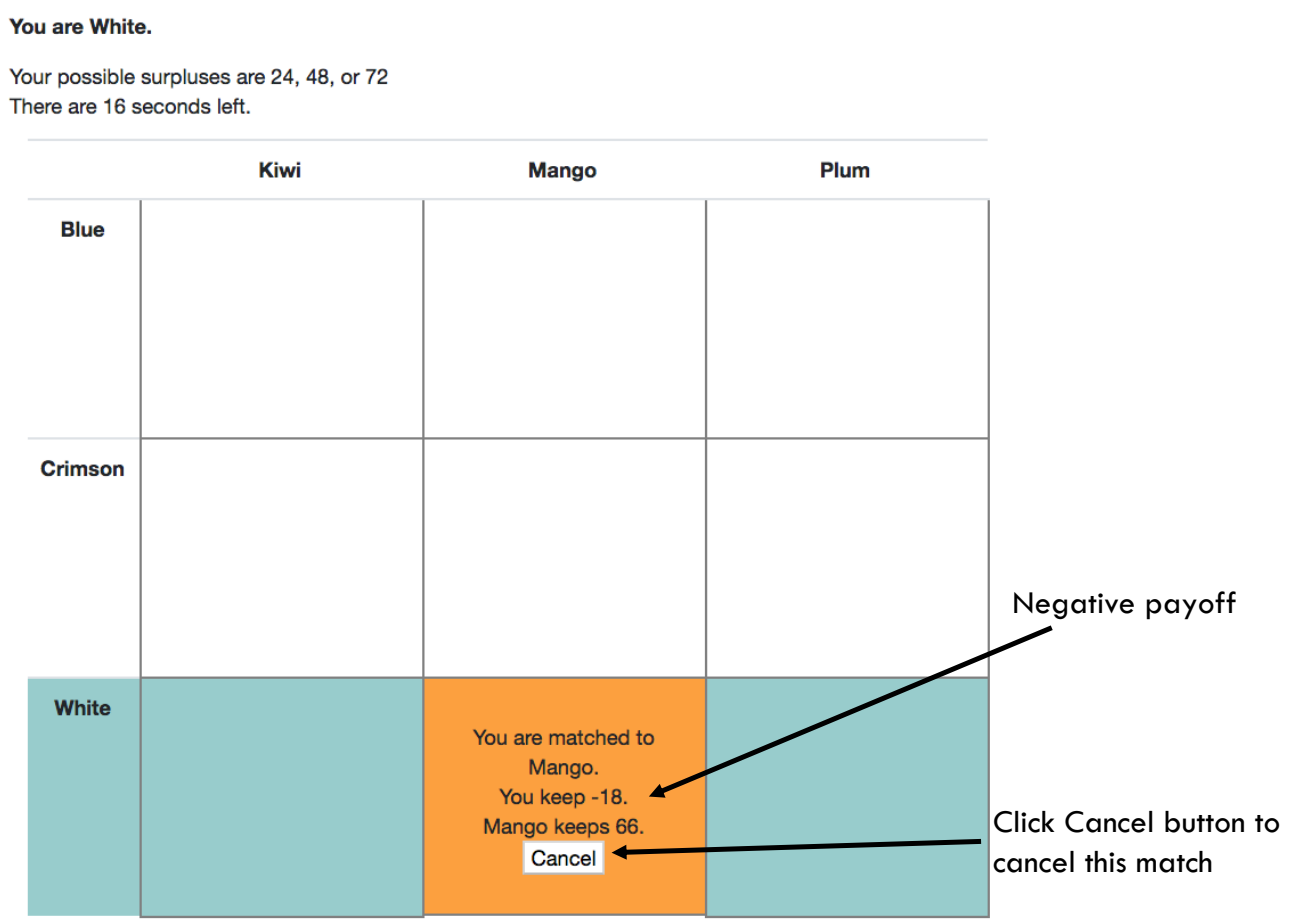

If you are currently matched, you can still make a match proposal to another individual. If the match proposal is accepted, then your current match will be broken. If you are currently matched, you can still receive match proposals from other individuals. If you accept a match proposal, then your current match will be broken. When you accept a match, or someone else accepts a match with you, any proposal you had made or received before will be automatically rejected. 
You are Blue.

Your possible surpluses are 16, 32, or 48

There are 14 seconds left.

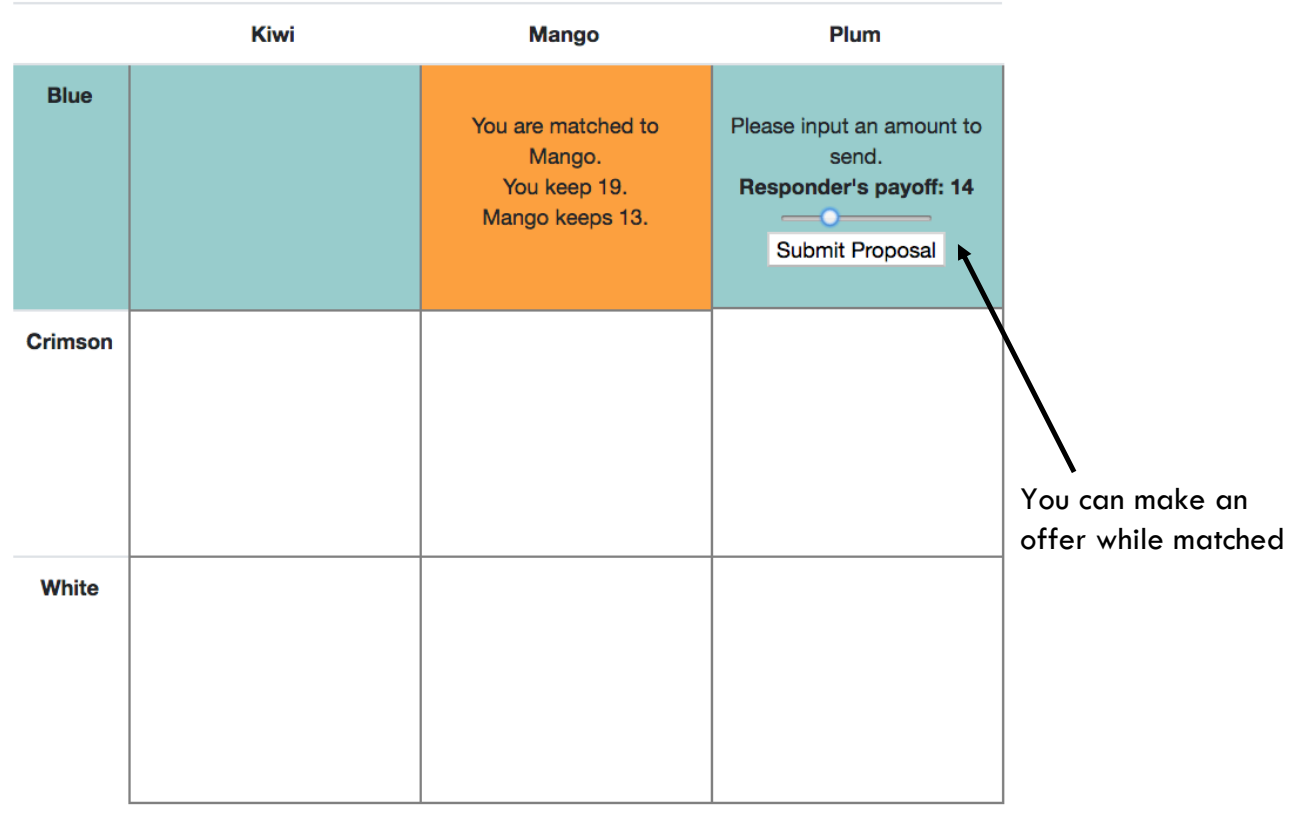


You are Blue.

Your possible surpluses are 16,32 , or 48 There are 26 seconds left.

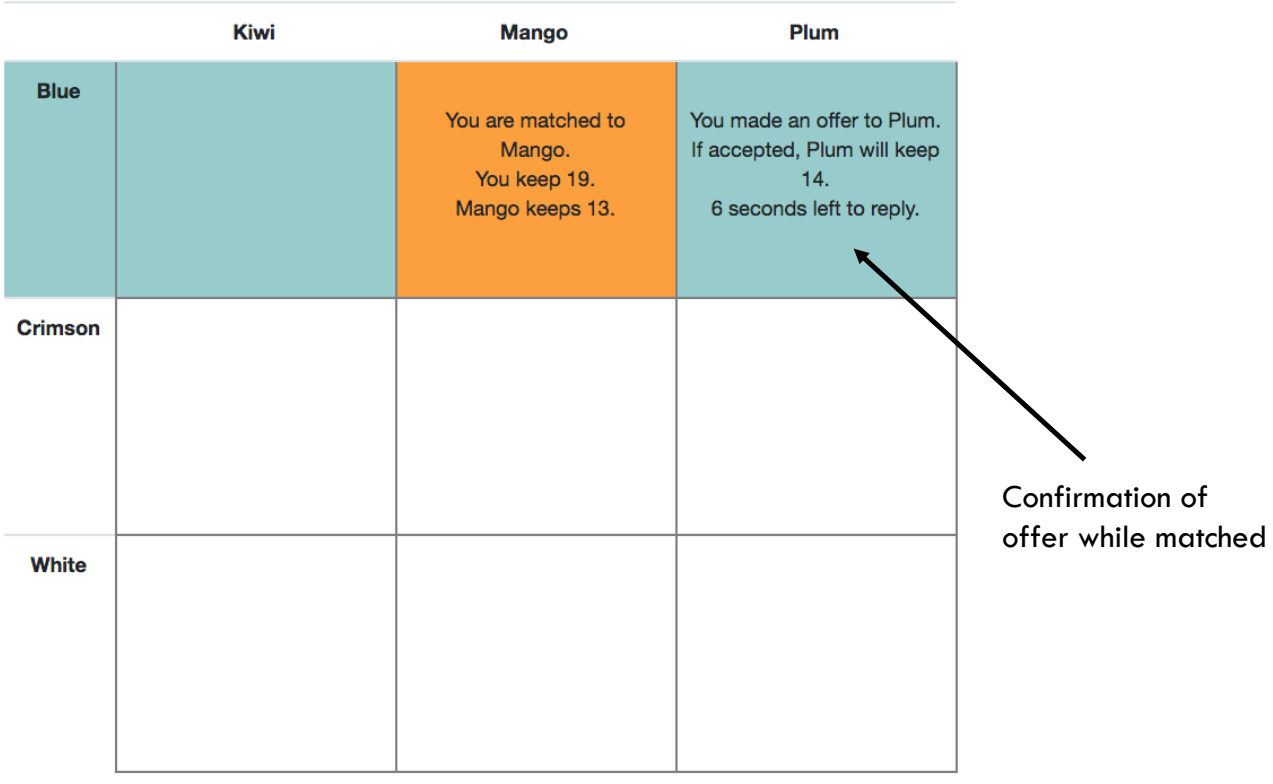


A round ends after 30 seconds of inactivity (i.e., after 30 seconds in which no new match proposals have been made). Whenever you are ready to proceed, you can also press the "Move on to Next Round" button. When at least 5 in your group press the button, you will automatically move to the next round (without waiting the 30 seconds). If any activity occurs after you press the button, the button will get released.

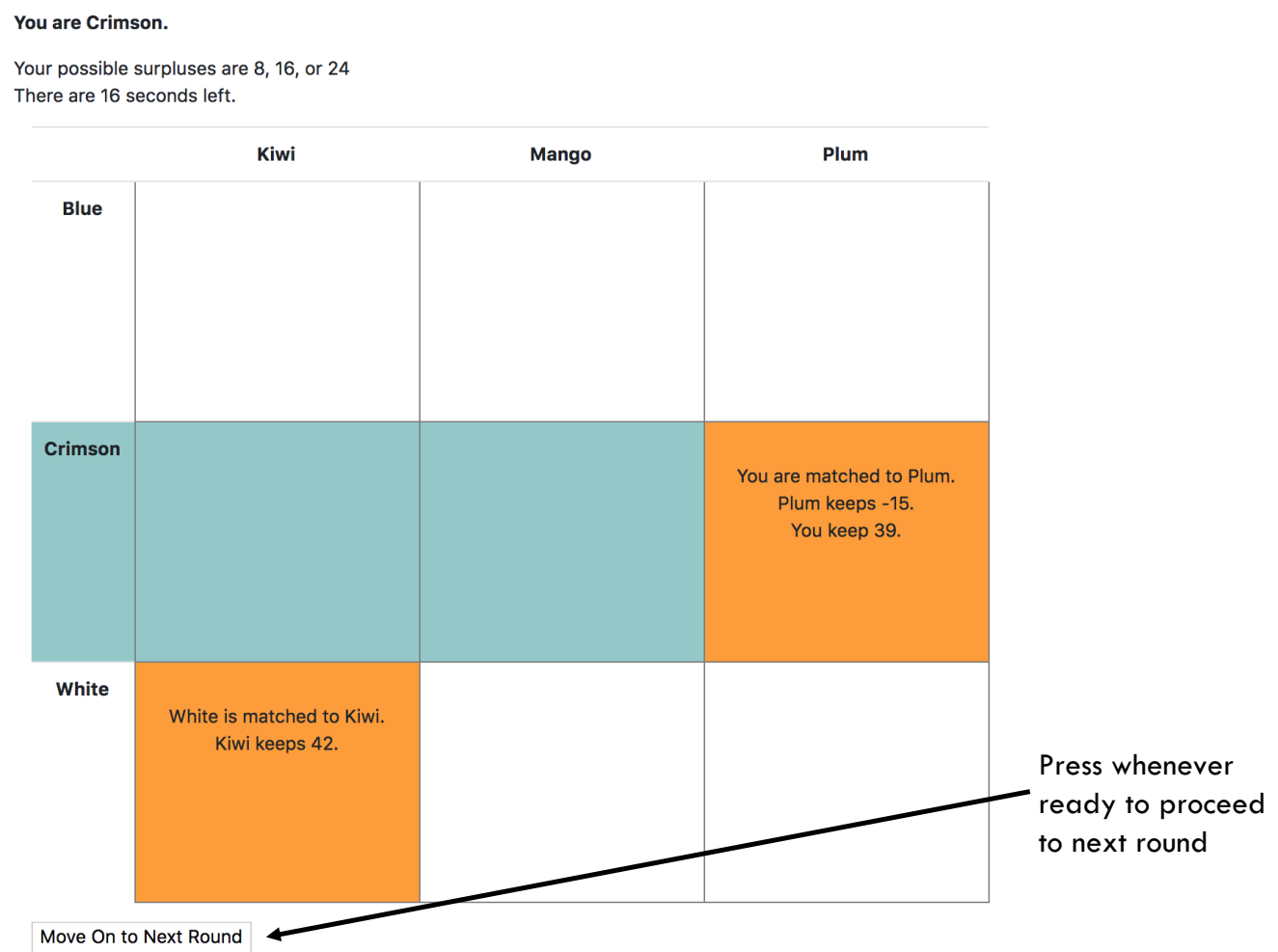


Payoffs for a Round. Your payoff when the round ends is your final payoff for that round. If you are not matched at the end of the round, your final payoff for that round is 0. Your payoff from temporary matches that are made and then broken during a round do not count for your final payoff.

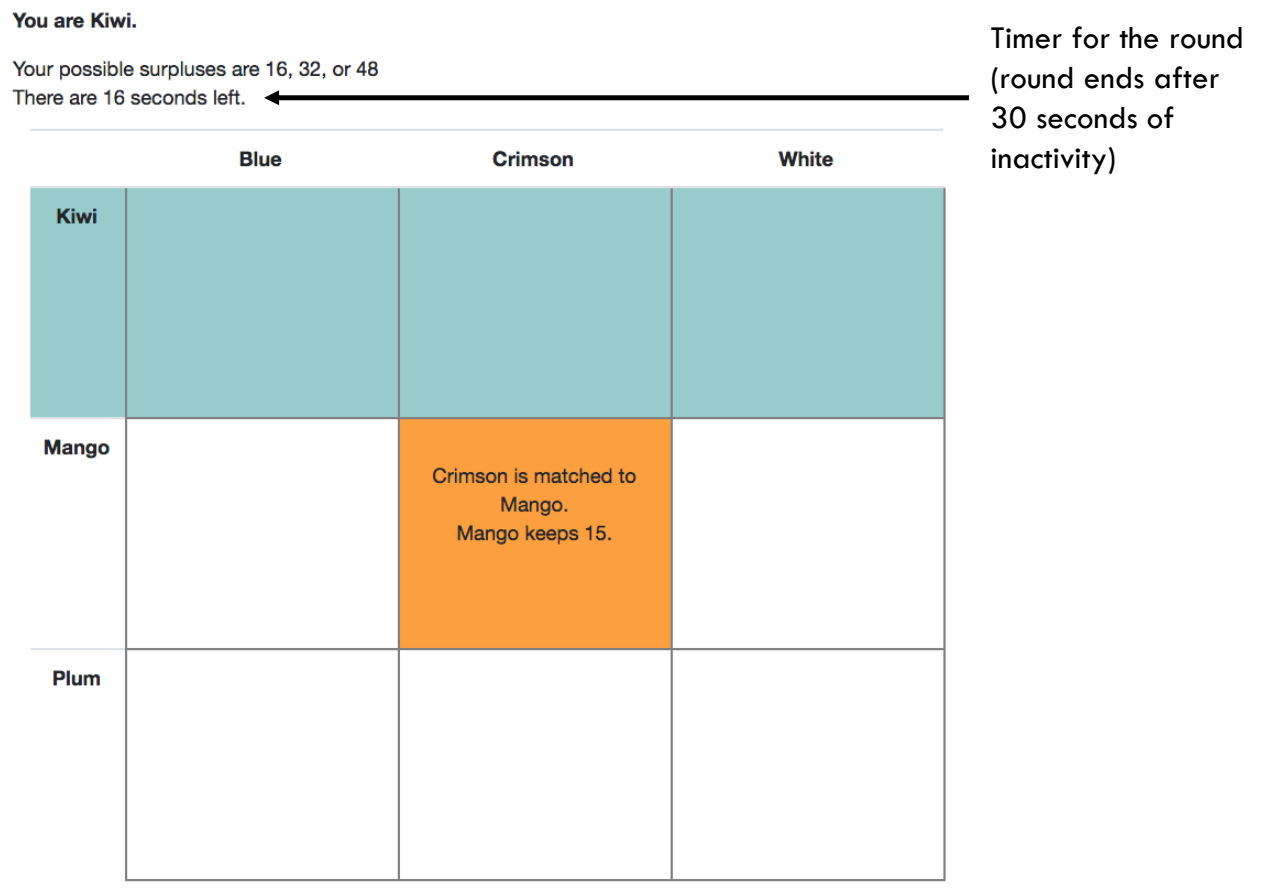

Post-Experiment. At the end of the experiment, you will be paid the sum of your payoffs across rounds (excluding the practice round). You will also be asked to complete several simple tasks at the end. You can earn additional money based on your decisions in these tasks.

Your Earnings. Your total earnings in the experiment are the sum of the following amounts:

- \$10 show-up payment

- payoff from 10 main rounds: 10 tokens = 1 dollar

- payoff from the simple tasks: 
You need not tell any other participant how much you earned. If there are no questions, we will now begin the actual experiment. 